Rabaska

Revue d'ethnologie de l'Amérique française

\title{
Les États-Unis et la Blanche Biche
}

\section{Francine Brunel-Reeves}

Volume 2, 2004

URI : https://id.erudit.org/iderudit/201646ar

DOI : https://doi.org/10.7202/201646ar

Aller au sommaire du numéro

Éditeur(s)

Société québécoise d'ethnologie

ISSN

1703-7433 (imprimé)

1916-7350 (numérique)

Découvrir la revue

Citer cet article

Brunel-Reeves, F. (2004). Les États-Unis et la Blanche Biche. Rabaska, 2, 51-89. https://doi.org/10.7202/201646ar d'utilisation que vous pouvez consulter en ligne.

https://apropos.erudit.org/fr/usagers/politique-dutilisation/ 


\section{Les États-Unis et la Blanche Biche}

Francine Brunel-ReEves

Montréal

\section{Traces d'un possible emprunt de la Blanche Biche par des Amérin- diens de la Caroline du Nord}

D'entrée de jeu le lecteur doit être informé que la première partie de ce travail s'appuiera sur plusieurs documents tirés de la toile, sources délicates à manier et souvent peu fiables, mais fournissant malgré tout de précieux renseignements à ceux qui, comme l'auteur de ces lignes, ont dû beaucoup s'y référer pour cause de difficultés temporaires de mobilité.

Dans l'état actuel des connaissances, on ne semble pas avoir rencontré aux États-Unis le schéma narratif de la complainte de la Blanche Biche qui circule dans le répertoire francophone traditionnel. Ce fait ne laisse pas d'intriguer, d'autant plus que la complainte est depuis fort longtemps présente en Acadie. On aurait pu imaginer que les Acadiens déportés tout le long des côtes étatsuniennes jusqu'à la Louisiane, après un détour par le Centre-Ouest de la France pour certains, l'auraient apportée dans leur bagage, tout comme plus tard les Québécois de l'estuaire du Saint-Laurent émigrés aux ÉtatsUnis au $\mathrm{XIX}^{\mathrm{e}}$ siècle. Pourtant, à ma connaissance aucune collecte sérieuse ne semble avoir déniché de Blanche Biche chez nos pourtant proches voisins.

Une « recherche Google avancée » de blanches biches ou blancs cerfs par mots-clés français et anglais, qui scrute toute la toile mondiale - je n'ai pas réussi à concentrer cette recherche uniquement sur les États-Unis ramène les étonnants résultats suivants, dans plusieurs langues (ces résultats fluctuent d'un jour à l'autre) :

" Google a recherché "blanche biche" sur la toile [en recherche avancée] et trouvé environ 514 résultats (16,500 résultats en recherche simple).

" Google a recherché "white doe" sur la toile [en recherche avancée] et trouvé environ 3,730 résultats $(833,000$ résultats en recherche simple) ». 
" Google a recherché "white hind" sur la toile [en recherche avancée] et trouvé environ 2,160 résultats $(315,000$ résultats en recherche simple) $){ }^{\prime}$

« Google a recherché "blanc-cerf' sur la toile [en recherche avancée] et trouvé environ 45 résultats (44 en recherche simple) ». Mais si on cherche "blanc cerf » sans trait-d'union on obtient 36000 résultats.

" Google a recherché " white deer » sur la toile [en recherche avancée] et trouvé un total d'environ 53,000 résultats (et un fabuleux $2,650,000$ résultats en recherche simple) ».

Est-il besoin d'insister ? Nous sommes tous au fait à présent qu'une courte campagne de pêche sur la toile peut ramener une énorme quantité de poissons, que des blanches biches il y en a beaucoup de par le monde, et il y en a pour ainsi dire partout, sous différentes formes qui s'équivalent dans l'imaginaire des populations (biches, daines, chevrettes, femelles du renne ou de l'élan, antilopes, gazelles, etc.). Bénéfiques ou maléfiques, bêtes sacrées ou surnaturelles, souvent tendres et innocentes victimes, elles foisonnent dans les croyances et superstitions, les lais, contes, légendes et complaintes traditionnelles, les œuvres d'art, en français, en anglais, dans toutes les langues d'Europe, en arabe, dans les contes africains, asiatiques (cf. site web http:// www.artandposters.com/byimage/OWPD0511L.htm, artandposters.com : "Cerf et blanche biche ", auvre japonaise, $\mathrm{XVI}^{\mathrm{e}}$ siècle, artiste anonyme ["Stag \& White Hind" - 16th Century Japanese]. Aucun détail n'accompagne cette superbe reproduction ; on ignore à quel élément de culture et de civilisation japonaise du $\mathrm{XVI}^{e}$ siècle cet artiste anonyme fait référence, mais la biche blanche avait certainement assez d'importance pour qu'il en effectue une représentation artistique à côté d'un cerf de couleur normale.)... et j'en passe. La mythologie et l'algorythmie « blanche biche » se côtoient sur la toile, aussi bien que des hôtels bien réels, des auberges, des restaurants, des parcs... sans compter de vraies blanches biches bien vivantes et photographiées - parfois isolées, mais parfois gambadant au milieu de grandes hardes de

1. En anglais on dit souvent White Hind pour Blanche Biche. Voici la définition de hind donnée par le Webster's New World Dictionary of the American Language (ci-après : WNWDAL), Second College Edition, The World Publishing Company, New York and Cleveland, 1970. Hind, nom, pluriel hinds, hind [traduction : Moyen anglais, dérivé de l'Ancien anglais, proche du germanique hide, dérivé de la base Indo-Européenne *kem-, qui n'a pas de cornes (appliqué aux espèces animales cornues), d'où le grec kemas, jeune cerf] : la femelle du cerf rouge, dans ou après sa troisième année] trad. FR. 
mâles et de femelles tous plus blancs les uns que les autres ${ }^{2}-$. Nous savons aussi que les mêmes informations peuvent se retrouver sur plusieurs sites différents, que d'autres sites n'ont rien à voir avec les mots clés, et que pour bien pêcher il faut d'abord apprendre à naviguer.

La recherche «Blanche Biche » en français est répétitive et rapporte en général beaucoup d'annonces d'enregistrements sur disques compacts, ou seulement les paroles ou la mélodie de la complainte francophone, le plus souvent celles de la version que j'appelle « Doncieux/Davenson " ${ }^{3}$, ou «DD "; aussi des contes avec différents types de récits, soit celui de la complainte, soit des remaniements poétiques ou littéraires, des récits de type arthurien, moyenâgeux ou de mythologie chrétienne (Geneviève de Brabant, Saint Gilles, etc.), plus quelques études de folklore et de mythologie.

La recherche "White Doe » de son côté, plus riche, balaye une grande partie des sites étatsuniens, rapportant aussi quelques chansons, de nombreux contes amérindiens ou traditionnels anglophones, ainsi que plusieurs poèmes et contes littéraires, plus un très grand nombre de sites soit d'hébergements hôteliers, soit touristiques (White Doe Inn, White Doe ou White Deer Lodge, etc.). J'avoue humblement n'en avoir pas encore fait le tour complet, et je ne m'aventurerai même pas à tenter de décrire les sites Blanc-cerf et White Deer tant ils sont nombreux.

Parmi les sites « White Doe » cependant, un conte amérindien provenant de la Caroline du Nord a particulièrement attiré mon attention. On peut en consulter plusieurs variantes sur la toile. Il s'agit de la célèbre « Légende de Virginia Dare » («The White Doe - Virginia Dare »). J'ai choisi d'en présenter

2. William Chapman et Alan E. Bessette, petit encart en page de fin, National Geographic Magazine, mai 1993 : Dans le nord de l'État de New York aux États-Unis, dans la région des Finger Lakes, on peut voir et admirer un troupeau d'environ 600 cerfs, biches et faons complètement blancs pour la plupart, qui n'ont pas les yeux roses et ne sont pas albinos. Il y a une cinquantaine d'années l'armée américaine avait enclos 12,000 acres de terrain pour y établir un dépôt (The Seneca Army Depot), et un petit groupe de cerfs de Virginie s'y est trouvé enfermé, dont certains présentaient un début de mutation vers le blanc. Ces animaux s'y sont reproduits et développés en milieu fermé, donc en situation de consanguinité tous azimuts, tant et si bien que, cinquante ans après, plus de la moitié des animaux sont complètement blancs et sont devenus un superbe sujet d'étude scientifique entre autres pour les chercheurs de l'Université Cornell. Ils sont même trop nombreux pour la dimension du dépôt, et on doit régulièrement en éliminer, de préférence ceux qui ne sont pas blancs, pour maintenir la population à un niveau adapté à la dimension du terrain. (référence transportée au début de la note) - Voir aussi les sites suivants : www. pressrepublican.com/outdoors/lib/2003/03162003out1.htm, Dennis April, «Is there a future for the Seneca Army Depot white deer », Press Republican, Plattsburgh, N.Y. le 3 mars 2003 ; et www.winecountry cabins.com/to_do/wildlife/deerwhite.htm. "White Deer in Finger Lakes ", sur le site de Wine county Cabins du lac Seneca.

3. F1894-99-1904a : version critique établie par Doncieux, publiée 2 fois, dans : 1. Georges Doncieux, Mélusine VII, 1894-95 ; 2. Romancero populaire de la France, Paris, Bouillon, 1904, Blanche Biche, pp. 237-242. Reproduite par Henri Davenson, alias Henri-Irénée Marrou, dans Introduction à la chanson populaire française, le livre des chansons, s'ensuivent cent trente-neuf belles chansons anciennes, Neuchâtel, Éd. Baconnière Boudry, “coll. Cahiers du Rhône », 1946, pp. 196-199. 
ci-après une variante qui me paraît assez représentative de cette étonnante histoire, dans une traduction de mon fait. On pourra consulter le texte anglais d'origine sur la toile indiquée en en-tête du conte :

«The White Doe - Virginia Dare »

Extrait de «Tales from the Coast! " (« Les contes de la côte »), Source : Clairbonrne S. Young, Guide de croisière pour les côtes de Caroline du Nord, Manteo, et l'île Roanoke www.icw-net.com/tales/mantwdœ.htm ${ }^{4}$

Plusieurs grands contes sont nés du mystère entourant la "Lost Colony » (= la Colonie Perdue). Virginia Dare est le sujet d'une légende particulièrement poignante qui s'est transmise en de multiples variantes. Selon la légende, les colons de Roanoke furent attaqués par des Amérindiens hostiles. Le Chef Manteo, revenant d'une expédition de pêche, arriva au beau milieu de l'attaque. Grâce à un tunnel secret il put conduire tous les habitants jusqu'à des canots qui étaient à proximité. Un voyage d'une nuit sur la rivière Pamlico $^{5}$ les amena à Hatteras, au village de Manteo. Les colons y furent accueillis par la tribu comme des frères et des sœurs.

Dès le début la blonde Virginia Dare à la peau si blanche parut merveilleuse aux yeux des Amérindiens. Au fur et à mesure qu'elle grandissait en taille et en âge, plusieurs braves jeunes gens lui firent la cour afin de l'épouser. La blonde jeune fille les aimait tous, autant les Amérindiens que les blancs, mais n'était pas prête encore à se choisir un mari.

Chico, l'homme-médecine de la tribu, était absolument envoûté par les charmes de la jeune fille. Bien que Virginia Dare fût très gentille avec lui, il était clair qu'elle ne ressentait pas pour lui la même ardeur, tant et si bien que, fou de passion, Chico jura qu'elle n'appartiendrait à aucun autre homme puisqu'elle ne voulait pas l'épouser. Faisant appel aux pouvoirs des nymphes de la mer, Chico attira Virginia sur l'île de Roanoke où elle fut transformée en biche blanche comme la neige dès qu'elle mit pied à terre.

Très vite on murmura qu'une biche blanche était la «meneuse » de tous les cerfs sur l'île Roanoke. Partout où allait la remarquable créature, tous les autres cerfs suivaient. Plusieurs grands chasseurs tentèrent de tuer cette créature mythique, mais aucune flèche ne semblait pouvoir la toucher. Avec le temps la biche blanche devint une légende, et en même temps un grand défi...

Finalement on organisa une grande chasse, et tous les jeunes braves de sang noble y vouèrent leurs efforts. De nombreux prix et marques d'honneur devaient récompenser celui qui tuerait la biche blanche. Le jeune Wanchese, fils du chef Wanchese, qui avait voyagé en Angleterre, possédait une flèche à pointe d'argent, que la reine d'Angleterre avait présentée à son père. Il lui

4. Le site suivant: www.usscouts.org/stories/s_deer.htmlhttp a mis en ligne une autre version de ce conte, beaucoup plus travaillée et littéraire. On en trouvera plusieurs variantes sur divers sites, assez courtement résumés.

5. Le plus ancien village de Caroline du Nord est dans la région de Pamlico/Neuse, qui comprend les communes sises sur le haut des rivières Pamlico et Neuse. Ces rivières sont des affluents majeurs du Pamlico Sound. [Trad. FR] 
attribuait des pouvoirs magiques et pensait qu'elle l'aiderait à atteindre le gibier qu'il chasserait.

Le destin voulut que Wanchese aperçut la biche blanche comme la neige. Il la visa soigneusement, décocha son arme meurtrière... et la pointe d'argent réussit là où toutes les autres flèches avaient échoué : la biche s'écroula sur le sol. Le brave jeune homme s'empressa d'aller chercher sa proie, mais toute sa joie s'envola pour faire place à la consternation lorsqu'il entendit la biche murmurer faiblement mais clairement dans un dernier soupir : « Virginia Dare ».

Si fantaisiste que cette histoire puisse sembler, elle s'est transmise sous une forme ou sous une autre depuis les tout débuts de l'histoire écrite de la Caroline du Nord. La longévité de ce récit ne peut que susciter notre étonnement et notre émerveillement.

\section{Un événement historique authentique}

Cette légende a pris naissance et s'est développée suite à un événement historique authentique qui s'est déroulé aux États-Unis à la fin du XVI siècle sur l'île Roanoke, située dans ce qui s'appelait alors la Virginie, mais qui est à présent en Caroline du Nord, soit la naissance du premier enfant né de parents européens en terre d'Amérique en 1587.

Cet enfant, une fille, est née dans une colonie d'environ 120 personnes amenées sur l'île Roanoke par Sir Walter Raleigh, qui avait déjà tenté à deux reprises et sans succès de coloniser la Virginie. Le petit groupe était arrivé sur trois vaisseaux dont un, "The Lion », était commandé par le Capitaine John White, que Raleigh nomma Gouverneur de la colonie de Roanoke avant de repartir. La mère du bébé, Eleanor (White) Dare, épouse du colon « planteur » Ananias Dare, était la fille du Gouverneur White, et le bébé fut nommé Virginia Dare parce qu'elle était le premier enfant européen né en Virginie, colonie baptisée ainsi en l'honneur d'Élizabeth I ${ }^{\text {ère }}$, "The Virgin Queen » (= la reine vierge). La naissance eut lieu un mois après l'installation des colons dans l'île. ${ }^{6}$

Trois mois après cette installation, le Gouverneur White retourna en Angleterre afin d'y chercher renforts et provisions pour sa petite colonie, mais en partant il fit promettre à ses colons de lui laisser un signe quelconque permettant de les retrouver si jamais ils se déplaçaient ou s'il leur arrivait quelque chose avant son retour. Malheureusement, l'Angleterre entrait alors en guerre avec l'Espagne, et tous les gens d'importance avaient bien autre

6. «The 18 [of August] Elenor, daughter of the Governour, and wife to Ananias Dare one of the Assistants, was delivered of a daughter in Roanoak, and the same was christened there the Sonday following, and because this child was the first Christian borne in Virginia, shee was named Virginia " (Thomas Hariot (1560-1621), " servant to Sir Walter Raleigh ", A Briefe and True Report of the New Found Land of Virginia, Electronic Edition. Illustrated by White, John, fl. 1585-1593. Translated out of Latin into English by Hakluyt, Richard, 1552?-1616. Site http ://docsouth.unc.edu/nc/hariot/hariot.html. 
chose en tête qu'un petit établissement isolé et précaire en terre d'Amérique. White ne réussit à revenir que trois ans plus tard, en 1590. Quand il remit les pieds à Roanoke, la colonie avait disparu, depuis assez longtemps déjà d'après certains indices, et ne lui avait laissé qu'un seul signe, énigmatique, soit le mot «Croatoan » gravé sur un arbre écorcé. Les Croatoans (aussi appelés les Hatteras) étaient une tribu d'Amérindiens de l'endroit qui s'était montrée plutôt amicale envers les Anglais.

La petite colonie ne fut jamais retrouvée, et son destin demeure un mystère jusqu'à aujourd'hui. On l'appelle encore « The Lost Colony » (= La colonie perdue), et de très nombreuses publications et sites racontent cette histoire et en discutent en long et en large. On trouve même sur la toile la relation de cette aventure, rédigée en anglais d'époque par Thomas Hariot, adjoint de Sir Walter Raleigh ${ }^{7}$ ainsi que des tableaux représentant des scènes de vie à Roanoke, œuvres d'un aquarelliste célèbre d'alors, nommé John White, dont plusieurs pensent qu'il était le même homme que le Capitaine et Gouverneur John White, grand-père du bébé Virginia Dare.

Le sort de l'enfant, surtout, a enflammé les imaginations, qui s'en sont donné à cœur joie, la transformant en belle, mystérieuse et à vrai dire fantomatique héroïne. L'île Roanoke est à présent un site historique important du « Dare County » avec un ancien Fort, musée et Auberge (Inn) ; on peut y admirer une statue et des tableaux représentant Virginia Dare (cf. site web http ://www.outerbanks.com/manteo/history/vadare.htm : Sculpture de Virginia DARE par Louise LANDER, 1859 (Manteo/Roanoke Island ICW-NET (B) CoastalGuide). assister à une pièce de théâtre sur le drame de la « Colonie perdue », aller en croisière sur un bateau portant son nom, que s'est aussi attribué un groupe de chanteurs. (Google a recherché «Virginia Dare » sur le Web. Environ 16,800 résultats en recherche avancée $(383,000$ en recherche simple...).

\section{Nombreuses théories sur le sort de « La colonie perdue » : les « Croatoans » au XXI" siècle.}

Une nation amérindienne particulière habite toujours la région. Ces Amérindiens se disent les descendants de « La colonie perdue », et plusieurs faits troublants renforcent cette possibilité : ainsi, quand on rencontra les descendants des "Croatan Cheraw » 50 ans après les faits, ils parlaient anglais, pratiquaient la religion chrétienne, connaissaient l'existence des « livres et papiers qui parlent », et portaient à $75 \%$ les noms de famille des colons disparus, mais tous les témoignages ajoutent qu'ils étaient extrêmement

7. Thomas Hariot, op. cit. 
métissés. [...] Quelques-uns avaient même les yeux gris [...]. Ces descendants, qui se nomment eux-mêmes aujourd'hui les "Lumbees » d'après la Lumber River (= Rivière à Bois, ou à billots) qui traversait leurs terres traditionnelles, étaient de races si mélangées qu'on leur a toujours refusé le statut fédéral de tribu amérindienne. ${ }^{8}$ [...] Après avoir examiné toutes les attestations écrites et orales connues, un historien « nationalement reconnu », cité par Julia White qui ne l'identifie malheureusement pas, en vint à la conclusion suivante en 1891 :

Les Croatoans d'aujourd'hui se disent descendants de « La colonie perdue ». Leurs habitudes, leur caractère et leurs caractéristiques mentales portent des traces d'origines à la fois amérindiennes et européennes. Ils parlent un anglais d'il y a 300 ans, et leurs noms sont fréquemment ceux que portaient les colons disparus. Aucune autre théorie n'a été proposée sur leur origine et celle-ci, basée sur des évidences externes et internes, parait la plus logique et historiquement la meilleure. Si elle est rejetée, le critique devra alors expliquer autrement l'origine d'un peuple qui, après 300 ans, présente les caractéristiques, parle la langue et possède les noms de famille de la seconde colonie anglaise implantée dans le monde de l'ouest. (Traduction FR) ${ }^{9}$

Plusieurs autres théories ont été proposées depuis sur le destin des colons disparus, soutenant ou rejetant celle des Lumbees, certaines assez plausibles, d'autres plutôt fumeuses. Plusieurs manifestent malheureusement des opinions racistes. Je n'ai résumé que celle qui a le plus d'adeptes, mais à vrai dire aucune n'est vraiment entérinée par les historiens.

\section{Localisation géographique de l'île Roanoke en Caroline du Nord}

« L'île Roanoke est située entre la terre ferme de la Caroline du Nord et la barrière d'îles Atlantique. La région est très riche historiquement, surtout parce qu'elle fut le site de la première colonie britannique dans le Nouveau Monde. Cette colonie ne survécut pas, et on l'appelle encore de nos jours « La colonie perdue ». La ville de Manteo, qui porte le nom d'un amérindien de Roanoke,est la plus grande ville de l'île. On y trouve de nombreux sites historiques [...]. Wanchese est un petit village de pêcheurs situé dans la partie sud de l'île $»^{10}$ (Traduction FR). (cf. la carte expedia.com localisant « The Lost Colony » sur l'île Roanoke, au milieu du « Croatan Sound » entre les «Outer Banks » et la terre ferme des côtes de la Caroline du Nord. «The Lost Colony » est située sur la pointe nord de l'île. Distances : de Montréal :

8. Ce résumé est un montage d'extraits du site web http ://www.native-languages.org/lumbee.htm, Native Languages of the Americas : Lumbee (Croatan, Cheraw) Language, et du site de la note 9.

9. Extraits de : Julia WHITE, The Lumbees and the Lost Colony, §13, http://www.meyna.com/ lumbee.html.

10. Site web http ://www.insiders.com/outerbanks/map-roanokeisland.htm 
776 milles ; de New York : 417 milles ; de Washington : 278 milles. Pour obtenir cette carte exacte on devra copier/coller en entier la longue adresse web qui suit dans la case de recherche : http://www.expedia.com/pub/ agent.dll? qscr $=$ mrdt $\& I D=3 X N s F . \& C e n P=35.932611,-75.708358 \&$ Lang $=$ USA0409\&Alti $=150 \&$ Size $=656,532 \&$ Offs $=0,0 \&$ MapS $=0 \&$ Pins $=\mid 35.932611$,75.708358|1|The+Lost+Colony\%2C )."

\section{La Blanche Biche et Virginia Dare}

La ressemblance entre la légende de Virginia Dare et la complainte de la Blanche Biche, fascinante à plus d'un titre, ne peut qu'inciter à une exploration plus poussée des deux récits. Tout d'abord parce que les États-Unis sont notoirement absents du corpus des versions recensées de la complainte (73 versions de terrain recensées, 84 en incluant les onze versions critiques), comme ils le sont des sept contes présentant le même schéma narratif, probablement huit avec cette légende de Virginia Dare. Parmi ces contes de Blanche Biche, un seul parfaitement identifiable, en parler créole, a été recueilli en Amérique entre 1920 et 1925 par Elsie Clews-Parsons, plus précisément en Martinique dans les Antilles françaises ${ }^{12}$. Ensuite parce que, mis à part le très important motif d'inceste frère-sœur, elle présente presque tous les autres motifs essentiels de la complainte ou des contes de Blanche Biche. Elle est intrigante surtout parce qu'elle semble directement dérivée de versions européennes de ce récit.

Or en Europe, on n'a pas recueilli de Blanches Biches chantées ou contées en Angleterre. Les versions connues proviennent de Suède, du Danemark, de France, de Belgique sous forme d'une bande dessinée qui est en réalité une version critique, et de Slovaquie (une chanson très ancienne et fragmentaire, sans motif d'inceste). Pourtant le seul pays d'Europe mentionné dans la légende de Virginia Dare est l'Angleterre, d'où provenaient les colons de Roanoke et la flèche à pointe d'argent, cadeau de la reine à l'Amérindien Wanchese qui l'avait rapportée en Amérique, flèche magique seule capable de faire tomber la biche blanche. Les îles Britanniques regorgent

11. Voir aussi : http://www.visitob.com/main/map.htm

12. Elsie Clews-Parsons, Folk-lore of the Antilles, French and English, Part I. « Sœur la té tourné biche ». The American Folk-lore Society, G.E. Stechert and Co., New York, Agents, 1933. Printed by J.J. Augustin, in Glueckstadt and Hamburg, in Germany. (2 volumes). Vol. I, pp. 255-256. [Bibl. du Musée de 1'Homme, Paris. cote F 1619.5 / C 5 / P 26 / V.1 / M.]. Ce conte semble être l'amalgame créolisé d'une version française et d'une version danoise de la complainte, en 50 couplets, publiée par Svendt Grundtvig en 1854. On sait que les îles Saint-Thomas, Saint-Jean et Sainte-Croix ont été danoises pendant 250 ans, et furent achetées par les États-Unis en 1917. En 1733, sur l'île Saint-Jean, les esclaves se révoltèrent contre les planteurs et possédèrent littéralement l'île pendant six mois. En désespoir de cause les planteurs (danois alors) demandèrent une aide militaire au gouverneur de la Martinique, qui leur envoya 400 soldats - français évidemment, à cette époque - qui réussirent à mater les révoltés. Voilà probablement comment les récit français et danois de Fille-Biche se sont retrouvés entremêlés (National Geographic Magazine, «The American Virgins » by DuBose Heyward and Daisy Reck, sept. 1940, pp. 289-290). 
de blanches biches toutes aussi surnaturelles les unes que les autres, mais dans d'autres types de chansons ou de récits, et jusqu'à présent on n'y a pas recueilli de récit identique à celui de la complainte.

Pour ce qui est de l'Amérique, outre le conte créole de la Martinique, on a recueilli au Canada francophone 45 versions chantées de la Blanche Biche, soit 25 au Québec et 20 en Acadie, mais aucun conte basé sur le même récit, du moins pas à ma connaissance. Et aucun récit identique reconnu comme tel n'a fait surface aux États-Unis.

Procédons maintenant, par le biais du tableau présenté ci-après, à une comparaison des motifs essentiels - qui seront commentés plus loin - entre la légende de Virginia Dare et une version québécoise de la complainte de la Blanche Biche choisie, parce que c'est de cette version qu'il sera question dans la seconde partie de ce travail ${ }^{13}$. Cette version présente le même schéma narratif et les mêmes motifs essentiels que les autres versions du corpus. Dans ce tableau, chaque récit est divisé en motifs, désignés par des lettres majuscules en caractères gras.

13. Version C1968a, provenant du Bas-Saint-Laurent au Québec, chantée dans la famille Lavoie/ Pouliot, de Nazareth, Rimouski. Yolande Rhéaume l'a recueillie une première fois en 1968 auprès de Mme Stella Pouliot-Proulx, quatrième des six enfants, tous chanteurs, d'Émile Pouliot et Louise-Anna Lavoie, et j'ai effectué de mon côté trois enregistrements en 1993 (enquête Reeves/Bodin), 1994, 1997 (enquête Reeves/G. Breton), auprès de la même informatrice et d'une de ses sœurs, $\mathrm{M}^{\mathrm{mc}}$ Jeanne d'Arc Pouliot-Lepage, plus un tournage-vidéo en mai 1998 (enquête Reeves/De Grosbois). 


\section{La Blanche Biche}

Version de la famille Lavoie/Pouliot, de Nazareth, Rimouski, recueillie le 15 novembre 1968 auprès de $M^{\text {me }}$ Stella PouliotProulx. Université Laval, Archives de folklore et d'ethnologie (AFEUL), coll. Yolande Rhéaume, Bob. 3-A, enr. 422.

A (séquence introductive) et B (refrain) :

1. C'est la fille d'un roi qui s'appell' Marguerite,

Elle est dedans un coin qui pleure, qui soupir'(e).

R. Et allez, allez dans le bois, dans le bois, ma mie.

2. Sa mère lui demande :-

Qu'avez-vous Marguerit(e) ?

C

3. - Vous souv'nez-vous ma mèr' le jour où je suis né(e)?

4. Vous m'avez fait laver dans un bassin doré,

5. Vous avez fait ch'ter l'eau dans le jardin des fé(es)...

6. Les fées m'ont donné un don, le jour où je suis fill(e)

7. Le jour où je suis fille, et la nuit Blanche Biche,

D

8. La chasse est après moi, les princes et les seigneurs,

9. Et mon frère Gérard qui-en vaut autant que quatre,

10. Allez lui dir', ma mère, allez lui dir' qu'il cesse !

11. - Cesse, cesse Gérard, c'est ta sœur Marguerite !

12. - Ma mèr', je n'comprends rien de ce que vous me dites

\section{Légende de Virginia Dare}

(sans le paragraphe d'introduction)
$\S \mathbf{A}$ (séquence introductive) et $\mathbf{B}$ (absent) : Dès le début la blonde Virginia Dare à la peau si blanche parut merveilleuse aux yeux des Amérindiens.

Au fur et à mesure qu'elle grandissait en taille et en âge, plusieurs braves jeunes gens lui firent la cour afin de l'épouser. La blonde jeune fille les aimait tous, autant les Amérindiens que les blancs, mais n'était pas prête encore à se choisir un mari.

§C. Chico, l'homme-médecine de la tribu, était absolument envoûté par les charmes de la jeune fille. Quoique Virginia Dare fût très gentille avec lui, il était clair qu'elle ne ressentait pas pour lui la même ardeur, tant et si bien que, fou de passion, Chico jura qu'elle n'appartiendrait à aucun autre homme puisqu'elle ne voulait pas l'épouser. Faisant appel aux pouvoirs des nymphes de la mer, Chico attira Virginia sur l'île de Roanoke où elle fut transformée en biche blanche comme la neige dès qu'elle mit le pied à terre.

§ D. Très vite on murmura qu'une biche blanche était le chef de tous les cerfs sur l'île Roanoke. Partout où allait la remarquable créature, tous les autres cerfs suivaient. Plusieurs grands chasseurs tentèrent de tuer cette créature mythique, mais aucune flèche ne semblait pouvoir la toucher. Avec le temps la biche blanche devint une légende, et en même temps un grand défi... 
E (voir plus bas, dans la complainte, le repas après la chasse)
§ E. Finalement on organisa une grande chasse, et tous les jeunes braves de sang noble y vouèrent leurs efforts. De nombreux prix et marques d'honneur devaient récompenser celui qui tuerait la biche blanche.

$\S$ F. Le jeune Wanchese, fils du chef

13. Gérard prit son mousquet par trois coups, il tir(e)

Wanchese, qui avait voyagé en Angleterre, possédait une flèche à pointe d'argent, que la reine d'Angleterre avait présentée à son père. Il lui attribuait des pouvoirs magiques et pensait qu'elle l'aiderait à atteindre le gibier qu'il chasserait.

G

14. Et au troisième coup, la Blanche Biche est prise
§ G. Le destin voulut que Wanchese aperçut la biche blanche comme la neige. Il la visa soigneusement, décocha son arme meurtrière...et la pointe d'argent réussit là où toutes les autres flèches avaient échoué : la biche s'écroula sur le sol.

(voir $§ \mathbf{E}$ ci-dessus)

(E)

15. - Allez, allez ma mère porter à la cuisine

16. Et dites aux cuisinières qu'ell's la fassent bien cuire

17. Venez, venez Marguerite goûter à la cuisine

H

18. Mon frèr' $j$ 'suis à la table, la première mise

19. Mon corps est dans un plat, au milieu de la table

20. Mon cœur à la cuisine, cloué à un' cheville

I

21. Gérard prit son mousquet

( couteau), à la forêt s'enfuit

On retrouva son corps où tomba

Blanche Bich(e)

R. Et allez, allez dans le bois, dans

le bois, ma mie
§ H. Le brave jeune homme s'empressa d'aller chercher sa proie, mais toute sa joie s'envola pour faire place à la consternation lorsqu'il entendit la biche murmurer faiblement mais clairement dans un dernier soupir : "Virginia Dare ".

§ I. (motif absent) 


\section{Détail des motifs}

A. a- Complainte : Une jeune fille se plaint de son sort qui est, on le verra plus loin, d'être poursuivie sous sa forme de biche blanche par des chasseurs (des « prétendants »), dont son frère. Cette chasse est nocturne dans 46 versions, et se déroule de jour dans vingt versions. Les autres versions omettent cette indication.

b- Légende : Une jeune fille ne veut pas de ses prétendants, dont aucun n'est son frère.

B. Refrain de la complainte : Isotopie du bois, non manifestée dans la légende, mais qui pourrait être implicite au vu des lieux où la chasse se déroule et en tenant compte de l'époque, bois et forêts étant l'habitat naturel de cette espèce de cervidé. N'y a-t-il pas d'ailleurs dans la région une rivière appelée « Lumber River » par les blancs, d'où les Lumbees tirent leur nom ? Les " Rivières à bois de coupe " coulent généralement au milieu des forêts puisqu'elle servent aux flottes de bois printanières qui suivent les hivers d'abattage des arbres.

C. Don des fées dans la complainte, ou des nymphes de la mer venant en aide à l'homme-médecine dans la légende, suivie de métamorphose en biche blanche dans les deux cas. Ce motif des fées responsables de la métamorphose n'apparaît spécifiquement que dans les versions canadiennes de la Blanche Biche. Dans les versions européennes, on rencontre des personnages équivalents deux fois en France, une fois au Danemark, dans le conte créole de la Martinique ${ }^{14}$, et des fées dans quelques contes français remaniés ${ }^{15}$. La

14. a) Version F1937a, une sage-femme, dans : Marguerite Gauthier-Villars Petit Chansonnier du Bourbonnais, Paris et Moulins, éditeur Gauthier-Villars 1937, avec mélodie et partition d'accompagnement pour piano. La Blanche Biche, chanson $N^{\circ} \mathrm{I}$, pp. 2-3; collectes effectuées dans un rayon de $20 \mathrm{~km}$ autour de Moulins et des environs de Bourbon l'Archambault.

b) Version Millien/Carroué, F1878a, une mauvaise femme dans : Achille Millien, dans Chants et chansons populaires du Nivernais, Paris, Leroux, 1906-1910 ; notation musicale de J G. Penavaire ; 3 vol., « La Fille-Biche » Vol. 1, p. 86-87. Rééd., Marseille, Jeanne Lafitte Reprints, 1981, augmentée d'un index des coupes et d'un index thématique, vol. 1, avec préface de Georges Delarue.

c) Version D1844a, une marâtre : Svendt Grundtvig, op. cit.

d) Conte créole de la Martinique : la marraine, dans Elsie Clews-Parsons, op. cit.

15. a) Récit sans motif d'inceste puisque c'est un prince amoureux qui chasse la fille-biche, dans «La biche au bois », par la Comtesse d'Aulnoy, dans Le Cabinet des Fées, publié entre 1785 et 1788 par le Chevalier de Mayer, 41 vol. • Réédition partielle, 1 volume, Arles, Éd. Philippe Picquier, 2000.

b) J.-Robert Colle, Contes et légendes du Poitou, illustrations de Henri Dimpre, collection des Contes et légendes de tous les pays, Paris, Nathan, 1951, pp. 62-73. Document communiqué par Jean-Loïc Le Ouellec. Amalgame de quatre légendes différentes, dont Le Sieur de Gallery, La Forêt de Chantemerle, La Blanche Biche, et l'Ours d'Angles.

c) Le conte de la Blanche Biche : Henri Pourrat, Le Trésor des contes, t. IX, Paris, Gallimard, 1958, pp. 246-251. Pourrat a repris différents motifs dont celui des fées du conte de $\mathbf{M}^{\mathrm{me}} \mathrm{d}^{\mathrm{A}}$ Aulnoy, auquel il a adjoint le motif d'inceste frère-soeur. 
blancheur de la fille-biche est soulignée avec plus d'insistance dans la légende amérindienne que dans la complainte.

D. Poursuite et chasse menées par plusieurs « nobles chasseurs », dont l'un est le frère de la fille-biche dans la complainte. Il n'est pas spécifié si la chasse se déroule de jour ou de nuit. Ici, dans cette version de la complainte comme dans la légende, cette chasse se déroule sans les chiens, qui sont présents dans presque toutes les versions de la complainte, lesquelles mentionnent habituellement plusieurs chiens, ce qui suggère une chasse à courre, sauf dans les versions scandinaves où le chasseur n'est accompagné que d'un seul chien ou chasse sans chien. L'absence de chiens dans notre version de la complainte pourrait être une simple défaillance de mémoire d'un informateur dans la chaîne de transmission ; pour ce qui est de son absence dans la légende, on pourrait supposer que les Amérindiens ne chassaient pas avec des chiens à cette époque, et que le motif leur aurait semblé superflu, possibilité qu'il y aurait lieu de vérifier. Peut-être encore, n'y avait-il pas de chiens dans le récit européen à l'époque où les Amérindiens l'ont intégré dans leurs traditions, mais c'est peu probable tant ce motif est constant ; de plus, un chien accompagne déjà le frère-chasseur dans la version suédoise de 1570 .

E. Manifestations prévues après la chasse : "repas de chasse » ou « repas cannibale » dans la complainte ; prix et récompenses dans la légende. Une variante de la légende de Virginia Dare parle plutôt de fêtes et de célébrations ${ }^{16}$. Le repas cannibale, symbolisant si on peut dire la finalisation de la consommation amoureuse, est absent des versions scandinaves et de quelques versions francophones de la complainte, sans que cette absence porte atteinte pour autant au motif d'inceste qui reste bien manifesté dans le motif de la chasse et de la mise à mort avec une arme pénétrante.

F. La flèche à pointe d'argent pour faire tomber la fille-biche : on a dans la complainte la seule occurrence dans tout le corpus d'un mousquet comme arme de chasse ; la flèche simple, de son côté, n'est mentionnée que dans les plus anciennes versions européennes recueillies, toutes les deux en Suède en 1570 et en 1670 . Quant aux flèches à pointes d'argent plus spécifiquement, elles étaient d'usage royal et certainement aussi dans les milieux de haute noblesse en Angleterre à cette époque. Ainsi, on raconte que dans ce pays, au XVI $\mathrm{XI}^{\mathrm{e}}$ siècle,

16. www.usscouts.org/stories/s_deer.htmlhttp, variante de la Légende de Virginia Dare, référence déjà citée. 
[...] alors que la reine Élizabeth I était encore une princesse habitant au Bishop's Palace à Hatfield, [...] elle allait de Hatfield à la Chasse d'Enfield escortée d'une suite de douze dames en satin blanc chevauchant des palefrois allant à l'amble, et de 120 hallebardiers (? yeomen) à cheval et vêtus de vert, afin d'y chasser le cerf. À l'entrée de la chasse, cinquante archers en bottes rouges et bonnets jaunes l'accueillaient, armées de flèches forgées d'argent, et chacun lui présentait une flèche à pointe d'argent ornée de plumes de paon ${ }^{17}$. (Traduction FR)

Dans la légende, il est bien spécifié que la flèche à pointe d'argent avait été donnée à l'Amérindien Wanchese par la reine d'Angleterre elle-même, qui était justement Élizabeth $1^{\text {èr }}$ à l'époque des voyages en Amérique de Sir Walter Raleigh.

Dans un conte littéraire remanié, probablement français plutôt que canadien, intitulé « Le Chasseur aux Étoiles », mis en ligne sur la toile en 1999, inspiré de la complainte de la Blanche Biche à laquelle l'auteur nous renvoie, il est question de l'arc d'argent de Renaud, frère de la fille-biche, plutôt que d'une flèche ${ }^{18}$. Une autre version de la légende de Virginia Dare, remaniée et littéraire aussi, met en scène deux chasseurs rivaux dont l'un est amoureux de la jeune fille, et l'autre la déteste parce qu'elle est Anglaise. L'amoureux chasse avec une flèche à pointe de perle qui devrait ramener la biche à sa forme humaine, l'autre avec une flèche à pointe d'argent qui la fera mourir ${ }^{19}$.

J'ignore la fréquence, dans les répertoires traditionnels, du motif de la flèche magique seule capable d'atteindre la proie poursuivie, et tous les renseignements qu'on pourra me fournir sur le sujet seront accueillis avec gratitude. Mais j'aurais tendance pour ma part à relier un tel motif au grand chasseur de la mythologie avec sa flèche à trois étoiles qui ne rate jamais sa cible, étudié avec une érudition remarquable par Christian David dans le Bulletin de la Société de mythologie française (BSMF) en $1994^{20}$.

17. Cf. site www.grandheritage.com/Hotels/European/England/West_Lodge/westlodgehistory.htm, "West Lodge Park Hotel - History 》.

18. Voir le site pinpin.mardyck.org/textes/chasseur.html.

19. Sur le site suivant : www.cayuse-press.com/thj11/11 text23.html, LB Sedlacek, Two arrows and $a$ White Doe, 2002. «website created and maintained by Cayuse Press. The Horsethief's Journal Cayuse Press P.O. Box 66003 Burien, WA 98166-0003 USA (c) 2002-2004 by Cayuse Press All Rights Reserved ».

20. Christian David, « La Belle aux cheveux d'or et l'archer qui chasse avec son chien - des legs indo-européens dans les romans tristaniens ", BSMF $N^{\circ} 174-175$, 1994, pp. 009>025. Voir la deuxième partie de ce travail, intitulée « L'Archer qui chasse avec son chien », p. 13, §'Le motif de l'arme infaillible; p. $16, \S$ Nemrod, inventeur d'un arc et de flèches magiques ; $\S^{`}$ Le chasseur et son chien ; la flèche à trois étoiles ; etc. • Le site web2.uqat.uquebec.ca/astro/chroniques « La chronique à René ", en janvier et février 2003, répertoriait aussi les différentes mythologies qui se sont développées autour de la constellation Orion. 


\section{G. Motif d'inceste frère-sœur}

a) dans la complainte : malgré les avertissements qu'il reçoit avec incrédulité, le frère poursuit sa sœur, la tue avec une arme à feu (une flèche à pointe d'argent dans la légende), et se prépare à la consommer au cours du repas de chasse qui suivra. Toutes les versions chantées et contées francophones et scandinaves de la Blanche Biche comportent cette symbolique d'inceste frère-sœur, à trois exceptions près : 1) la version suédoise de 1670 , où c'est son fiancé qui chasse la fille-biche, dont la couleur n'est pas spécifiée $^{21}$;2) la version slovaque où c'est le garde-champêtre qui chasse la fille-biche, dont la couleur n'est pas spécifiée $e^{22}$; 3) la version Millien/Carrouéé ${ }^{23}$ où c'est le roi son père qui est le chasseur incestueux de la fille-biche, dont la couleur n'est pas spécifiée.

b) dans la légende : L'absence de ce motif dans la légende et son importance dans la complainte abolissent-elles toute possibilité de filiation entre les deux récits ? On sait que l'inceste est un tabou universel, particulièrement l'inceste frère-sœur - sauf très anciennement dans les familles royales, en Égypte, chez les Incas, etc. - , et que c'est un motif mythologique important dans un grand nombre de cultures. Si on en croit le botaniste finlandais Pehr $\mathrm{Kalm}$, les cultures amérindiennes avaient toutes horreur de l'inceste ${ }^{24}, \mathrm{du}$ moins celles qu'il a pu observer au cours de ses voyages en Amérique, ce qui pourrait expliquer son absence dans le récit amérindien. Elle pourrait résulter par ailleurs de l'absence du motif aux $\mathrm{XVI}^{\mathrm{e}}$ et $\mathrm{XVII}{ }^{\mathrm{e}}$ siècles dans des versions européennes, ou d'une omission, volontaire ou non, dans le processus de transmission de la part des Européens. Sa symbolique et la clarté de son énoncé dans la complainte ne sont pas évidentes à tout le monde, et encore maintenant plusieurs personnes ne les décèlent pas du tout. Peut-être échappaient-elles aux Européens qui auraient pu faire connaître le récit aux Amérindiens. C'est Thierry Charnay qui soulignera ce motif pour la toute

21. S1670Aa : Sveriges Meteltida Ballader - ci-après, SMB -, Svenskt Visarkiv, Stockholm, vol. I, 1983, p. 64, v.o. recueillie dans le Västergötland auprès d'Ingierd Gunnarsdotter. • Aussi publiée par Adolf Iwar Arwidsson dans Svenska Fornsånger, en samling af Kämpavisor, Folk-visor, Lekar och Dansar, samt Barnoch Vall-Sånger, Andra Delen, Stockholm, 1837, Tryckt hos P.A. Norstedt é söner, Kongl. Boktryckare ; pp. 260-262, “ Den Fortrollade Jungfrun 》, version B. - Cette version a été traduite et publiée en français par Léon Pineau dans la Revue des traditions populaires (RTP), 8-9, XIX, Paris, août septembre 1904, p. 328.

22. Ballades populaires slovaques, Prague, Artia, Tchécoslovaquie, Traductions de Charles Moisse, 1963, chapitre " Des temps très anciens ", p. 13. Chanson probablement fragmentaire, sans véritable dénouement ni mise à mort de la fille-biche, métamorphosée par sa mère, qui n'est pas blanche.

23. Version Millien/Carroué, F1878a, Achille Millien, op. cit.

24. Voyage de Pehr Kalm au Canada en 1749, traduction annotée du journal de route par Jacques Rousseau et Guy Béthune avec le concours de Pierre Morrisset. Montréal, Éd. Pierre Tisseyre, 1977, p. $616, \S 3$. 
première fois dans sa thèse sur la Blanche Biche en $1986^{25}$, après les nombreux commentaires des savants, chercheurs et autres érudits sur cette complainte depuis sa première attestation en France, en 1841, commentaires n'insistant pratiquement que sur les motifs de métamorphose et de lycanthropie.

Il existe en France un autre récit sans motif d'inceste, provenant de la Vendée dont le schéma narratif est très proche de la complainte et de la légende. Cité par Jean-Loïc Le Quellec dans La Vendée mythologique et légendaire $^{26}$, il relate un fait divers ancien et probablement authentique, transformé par la transmission orale en une légende étonnante de chasse à la biche qu'une stèle, « La colonne de la Demoiselle », rappelle encore de nos jours à la mémoire populaire:

La colonne dite de la demoiselle marquerait l'emplacement de la tombe de la Biche de l'Étenduère. C'était une « demoiselle » qui fut changée en biche par un Seigneur anglais dont elle avait refusé les avances. Depuis quelque temps donc, elle " courait », la nuit, en effrayant le pays, quand un habitant l'abattit d'un coup de fusil. Mais « il n'avait pas mis une balle bénite dans son fusil, alors il tua une biche ». S'il avait fait bénir sa balle, la fille aurait repris sa forme humaine en mourant mais, comme ce n'était pas le cas, et que le curé refusait de faire entrer cet « animal » dans les lieux consacrés, on décida de l'enterrer sous cette " colonne " appelée depuis la " Tombe de la Demoiselle ». Une plaque de marbre y aurait même été apposée, mais on dit qu'elle aurait été brisée par les colonnes infernales (L. Barbarit, Récit d'autrefois..., " La Biche de l'Étenduère ", Le Puyfolais, 1983, 17:32). ${ }^{27}$

Le motif d'inceste frère-sœur est donc absent de ce récit, et le responsable de la métamorphose est un Seigneur anglais, nationalité qui n'est pas à dédaigner dans nos interrogations sur la légende de Virginia Dare. La blancheur de la femme-biche n'y est pas spécifiée et n'est décelable que dans l'analogie du récit avec celui de la blanche et pure sainte Maria Goretti. Son dénouement inattendu a de toute évidence subi la forte emprise du monde clérical, comme dans vingt-sept versions françaises et canadiennes de la complainte ${ }^{28}: 1$ 'objet meurtrier aurait dû être une balle bénite par le curé, qui aurait joué le même rôle que la flèche magique à pointe d'argent si l'habitant

25. Thierry Charnay, op. cit. « La chasse, désir incestueux du frère » p. 169 ; « La relecture du récit comme celui d'un "inceste" entre frère et soeur » p. 170 ; «Le motif de l'inceste », p. 173.

26. Jean-Loïc Le Quellec, La Vendée mythologique et légendaire, Geste Éditions, 1996, « Les Herbiers, Biche de l'Étenduère ", p. 136.

27. Voir le site web http://www.villedesherbiers.com/Site-Herbiers/Culture-Loisirs/Tourisme/ etenduere/accueil-etenduere : Stèle de la Demoiselle, Photo : J. Vincent, 2003 (Légende de La Biche de l'Étenduère, Les Herbiers, (Pays de la Loire), Vendée, $85500 ; 13413$ habitants).

28. Dans la plupart de ces vingt-sept versions, l'énoncé de ce dénouement correspond à peu près à ceci : «Dans le royaume des cieux ma place y est promise/Mais au fond des enfers, la tienne y est acquise ». Un tel dénouement, sûrement d'insertion tardive dans la longue chaine de transmission du récit, annule son caractère tragique et fataliste pour le transformer en récit culpabilisant et moralisant. 
avait fait bénir ses balles avant d'entreprendre sa chasse, permettant du même coup à la victime de reprendre sa forme humaine en mourant... ce qui aurait été une bien mince consolation pour elle autant que pour Virginia Dare, on en conviendra ! Mais la balle n'ayant pas été bénite, la malheureuse est restée biche en mourant.

Un conte littéraire vendéen de Blanche Biche comporte la séquence suivante où il est question d'une flèche bénite qui fait tomber la fille-biche :

[...] Renaud se piqua au jeu et partit lui-même à la poursuite de l'insaisissable animal. Il l'aperçut plusieurs fois, fit voler dans sa direction une flèche rapide mais ne put jamais l'atteindre; il se passionna pour cette décevante poursuite au point d'en perdre le boire et le manger.

Il finit par se persuader que la bête était ensorcelée. Il se rendit en cachette à l'abbaye de l'Absie et demanda au prieur de bénir une flèche de son carquois. La nuit suivante, il se lança dans la forêt, découvrit des traces, s'approcha de la biche et, saisissant la flèche béni[t]e, banda son arc de toutes ses forces et tira. La bête, qui avait reçu le trait en pleine poitrine, se cabra avec un cri d'agonie. Une tache rouche étoila sa blanche poitrine. Renaud accourut pour le coup de grâce. Il fut ému par le visage de la biche qui tournait vers lui ses grands yeux remplis de larmes mais, détournant la tête, il lui planta sa lame dans le cœur. $[\ldots]^{29}$.

Dans la légende de Virginia Dare, on ne nous dit pas quel sortilège a doté la flèche à pointe d'argent de son pouvoir magique, mais ici la bénédiction d'un homme d'église s'y est substituée pour ensorceler la flèche de Renaud. Plusieurs ballades à motif d'inceste du domaine européen semblent comporter un motif parallèle, où la personne incestueuse attribue un pouvoir permissif aux bénédictions cléricales : dans une ballade grecque, la mère incestueuse propose à son fils d'aller voir un prêtre pour qu'il bénisse leur mariage ${ }^{30}$; ou le père incestueux va demander une sorte de " bénédiction/permission " à son curé ou pasteur pour épouser sa fille, permission accordée parce que le père a posé une question ambigüe $\mathrm{e}^{31}$.

Le récit de la Biche de l'Étenduère, sans motif d'inceste, rappelle fortement les légendes de garaches vendéennes, animaux blancs nocturnes - le plus souvent des chèvres blanches - qui viennent effrayer les gens en venant danser autour de chez eux la nuit, ou poser leurs museaux sur les rebords des

29. Jean-Robert Colle, "La Forêt de Chantemerle ", dans Contes et légendes du Poitou, Paris, Nathan, coll. "Contes et légendes de tous les pays ", 1951, pp. 62-73. Ce conte aligne à la suite les légendes du Sieur de Gallery, de la Forêt de Chantemerle, de la Blanche Biche, et celle de l'Ours d'Angles.

30. Brewster, The Incest Theme in Folksong, Helsinki, FFC 212, 1972, p. 10, deux versions; et note $30:$ « Plus de 40 variantes ont été enregistrées, la plupart provenant de l'ouest de la Grèce ». L'auteur n'a pas retenu la complainte de la Blanche Biche pour son étude, omission qui pourrait être due à sa méconnaissance du répertoire francophone traditionnel ou bien, comme beaucoup d'autres, il n'a simplement pas décelé le motif d'inceste dans cette chanson.

31. Ibid, p. 12, et note 36, p. 32, Sbornik za narodni umotvoreneniya, XXV, 133, no 51 . 
fenêtres. Ils sont souvent tués par des habitants de l'endroit, parfois même par le curé, et reprennent alors forme humaine, généralement celle de belles jeunes femmes, dont on apprend le lendemain, par un étranger venu à leur recherche, qu'elles venaient du Nord, ou d'Angleterre, ou d'un autre village.

Les convergences et divergences de ces récits avec ou sans motif d'inceste frère-sœur, biche de l'Étenduère, complainte et contes de Blanche Biche, et garaches, dessinent un tableau de bien proche parenté, sinon de filiation directe, qui aurait pu entrer en ligne de compte dans l'omission du motif d'inceste dans la légende de Virginia Dare.

H. Révélation de son identité féminine par la fille-biche : La fille-biche révèle verbalement son identité féminine, une fois morte et cuisinée dans la complainte, en mourant, dans la légende. Ce motif est présent sous une forme ou une autre dans toutes les versions complètes et dans tous les contes de Blanche Biche du corpus.

I. Suicide du frère : motif absent de cette version du conte amérindien, qui s'arrête à la découverte de la femme sous l'apparence de la biche, comme dans certaines versions de la complainte ; dans d'autres versions contées du récit, le chasseur désespéré va se perdre dans la forêt pour jamais. Ce motif du suicide, rare et qui paraît ancien, confère au récit un caractère tragique, fataliste, mais pas fondamentalement moralisant. Totalement absent des versions françaises de la complainte, il n'apparaît que neuf fois dans tout le corpus - qui comporte huit sortes de dénouements différents -, soit une fois en Suède et huit fois au Canada francophone (quatre fois au Québec et quatre fois en Acadie).

\section{Comment ce récit serait-il arrivé en Caroline du Nord ? Plusieurs possibilités.}

Si on en croit les écrits relatant la légende de Virginia Dare, ce récit est indatable et se perd dans la nuit des temps... étatsuniens à tout le moins. Entre la disparition de la "Colonie perdue » et aujourd'hui, 417 ans se sont écoulés : de quand date la première attestation de la légende, et qui l'a notée alors? On aimerait le savoir, mais la toile ne nous renseigne pas sur ce point. Souvenons-nous toutefois que sur notre continent la nuit des temps n'est pas aussi profonde que dans les vieux pays, et qu'une église, une armoire, un air ou un conte traditionnel de cent ans d'âge y sont souvent perçus comme des objets ou des faits de grande antiquité.

J'ai cru comprendre, d'après certains documents réfutant la théorie des Lumbees, qu'on a souvent cherché à rattacher le « mythe de la colonie perdue » aux anciennes cultures des vieux pays. Des folkloristes anglophones auraient pu tenter de relier aussi la légende de Virginia Dare au très vieux récit européen de la Blanche Biche, mais s'ils l'ont fait les documents que 
j'ai pu consulter - uniquement sur la toile et pas exhaustivement, je dois l'avouer -, n'en font pas mention.

Tout en étant peu documentée en traditions amérindiennes, qui n'ont pas fait partie jusqu'ici de mes sujets de recherche, je sais qu'elles sont riches de récits mettant en scène des animaux blancs, bisons, daims, cerfs, biches (où cerfs et biches blancs sont souvent désignés par des termes très poétiques tels : " Frosted doe », "Snow-white doe », " Ghost doe or deer », « Moon deer or doe » "Lily-white doe », sans compter la fameuse « milk-white doe » qu'on rencontre aussi bien dans les traditions anglophones qu'amérindiennes $^{32}$ ), etc. Mais il semble extrêmement plausible que la légende de Virginia Dare soit d'inspiration européenne. Si c'était le cas, comment ce récit auraitil pu arriver en Caroline du Nord et s'insérer dans les traditions amérindiennes de la région? Les possibilités suivantes peuvent être suggérées :

1. A supposer que notre récit de Blanche Biche ait eu cours dans les traditions anglophones des îles Britanniques aux $\mathrm{XVI}^{\mathrm{e}}$ et $\mathrm{XVII}{ }^{\mathrm{e}}$ siècles sous forme de «ballade » ou de conte qui aurait disparu du répertoire anglophone depuis, elle aurait pu arriver avec des colons venus de là. Pas nécessairement ceux de la " Colonie perdue ", mais ceux qui sont venus s'établir - avec succès cette fois - en Virginie et dans les Carolines au XVII ${ }^{\mathrm{e}}$ siècle ou plus tard. Il serait curieux cependant qu'elle n'ait pas laissé de traces précises et reconnaissables dans le répertoire des îles Britanniques, et que les grands folkloristes anglophones, Francis James Child par exemple, qui connaissait bien la Blanche Biche dans ses versions françaises et scandinaves, et l'avait même commentée dans son introduction à la ballade Leesome Brand — n'en ait pas eu connaissance ${ }^{33}$.

2. Une seconde possibilité est qu'un ou plusieurs colons anglais de cette période aient connu une version française chantée ou contée de la Blanche Biche, qui a été plusieurs fois recueillie en Normandie au XIX ${ }^{c}$ siècle, dans la péninsule du Cotentin entre autres, pas très loin des côtes d'Angleterre après tout. Quelques coïncidences de dates et des substitutions de prénoms dans la complainte suite à des événements historiques marquants laissent croire qu'elle se chantait déjà dans les départements d'Ille-et-Vilaine et de l'Orne sous Henri IV, vers 1603 ou peu après, et que même un de ces événements - qui sera le sujet d'un autre article - a eu suffisamment de

32. Traductions: Biche de givre ou de frimas, Biche blanche comme la neige, Biche ou cerf fantôme, Cerf ou biche de lune, Biche blanche comme le lys, sans compter la fréquente Biche blanche comme le lait... etc.

33. Francis James Child, (1825-1896), The English and Scottish Popular Ballads, Part I. Boston, Houghton, Mifflin \& Co., New York / Cambridge / London, 10 volumes à l'origine, reliés en 5 volumes. Leesome Brand, Ballad n ${ }^{\circ}$ 15, vol. 1, versions A et B, pp. 177-184, plus vol. 5, p. 501-502, additions et corrections. $\bullet$ Cote : BN imprimés: $4^{\circ}$ OYK 18. 
retentissement en Angleterre pour avoir peut-être inspiré à John Ford une pièce de théâtre en 1633 et s'insérer en tant que motif dans un certain nombre de chansons traditionnelles anglophones et peut-être même scandinaves ${ }^{34}$.

3. Une troisième possibilité serait un apport suédois. Les Suédois avaient établi des postes de traite le long de la rivière Susquehannah entre 1633 et 1638 , sur des territoires qu'une carte nomme la Nouvelle-Suède et situe alors juste au-dessus de la Virginie en 1685, alors qu'ils avaient été conquis par les Hollandais dès 1665. Mais des descendants des pionniers suédois sont restés sur ces terres et, cent ans après, le botaniste finlandais Pehr Kalm relatait ses rencontres avec eux ${ }^{35}$. Deux versions de la complainte ont été recueillies en Suède, en 1570 et en 1670 , la seconde ne comportant pas le motif d'inceste frère-sœur ${ }^{36}$. Une petite objection à cette possibilité d'apport suédois serait que, dans les versions scandinaves, la biche n'est jamais blanche et sa couleur n'est pas spécifiée.

4. Elle aurait pu aussi arriver directement de France, sous la forme qu'on lui connait, avec des ressortissants français, marins, soldats de passage, ou peut-être même des colons qui se seraient établis dans ces parages au XVII ${ }^{\mathrm{e}}$ siècle. Le texte suivant, tiré de la toile, confirme une telle présence de ressortissants français parmi les immigrants du XVII ${ }^{e}$ siècle en Amérique, entre autres des huguenots fuyant les persécutions ; on sait que plusieurs huguenots venaient du Centre-Ouest de la France, où douze versions de la complainte de la Blanche Biche et plusieurs contes ont été recueillis :

Mélange de cultures : La majeure partie, soit $90 \%$, des colons venant en Amérique au $17^{\circ}$ siècle, sont Anglais. Un petit nombre de Hollandais et Suédois et d'Allemands se sont établis dans la région intermédiaire entre le Nord et le Sud, et quelques Huguenots français dans les Carolines et ailleurs, des esclaves africains, principalement dans le Sud. Dispersés en petits groupes, vivent des Espagnols, des Italiens et des Portugais. Néanmoins, l'ensemble des colons d'origine non anglaise ne représente que $10 \%$ à peine du total.

34. Sous Henri IV s'est déroulée l'histoire authentique, tragique et fatale des amants incestueux de Tourlaville, Julien et Marguerite de Ravalet, fils et fille du Seigneur Jean de Ravalet, seigneur de Tourlaville près de Cherbourg. Les jeunes amants qui n'avaient pas vingt ans furent décapités tous les deux, en Place de Grève à Paris en 1603, devant une foule de badauds qui avaient suivi le déroulement de leur procès tout au long, comme aujourd'hui on regarde une série télévisée. Cette histoire eut tellement de retentissement que des ouvrages sur le sujet ont été publiés depuis le début du XVII' siècle jusqu'à la fin du XX⿳亠口冋口十 siècle.

35. Pehr Kalm, cité par Denis Delâge dans Le Pays renversé, Montréal, Boréal, 1985, pp. 259 262. L'auteur se réfère à l'édition anglaise : P. Kalm, Travels in North America, tome 1, pp. 266-268, 270, 285-286.

36. a) S1570Aa : Harald Oluffson Visbok 1570, Svenst Visarkiv, Stockholm : v.o. recueillie dans le district de l'Uppland.

b) S1670Aa: SMB, op. cit. 
Après 1680, un grand nombre d'immigrants viennent d'Allemagne, d'Irlande, d'Écosse, de Suisse et de France ; l'Angleterre cesse d'être la principale source d'immigration.

[...] La majeure partie des immigrants venus des autres pays que l'Angleterre, s'adaptent aux coutumes des premiers colons - ce qui ne signifie nullement qu'ils se transforment tous en véritable fils d'Albion. Ils adoptent, il est vrai, la langue anglaise, les lois et nombre de coutumes d'Angleterre; mais seulement dans la mesure où les conditions locales les ont modifiées. De ce creuset doit se dégager une civilisation unique en son genre, mélange de culture anglaise et européenne mais conditionné par l'environnement du Nouveau Monde ${ }^{37}$.

5. Une cinquième possibilité serait l'arrivée de la complainte ou du récit francophone avec des Acadiens, soit encore une fois marins de passage naviguant sur les vaisseaux, commerçant avec les colonies anglaises et les Antilles entre 1604 et la prise de l'Acadie par les Anglais, soit plus tard avec des Acadiens déplacés par la grande déportation, au milieu du XVIII ${ }^{e}$ siècle. D'après les statistiques sur la diaspora acadienne, on comptait 300 Acadiens déportés en Caroline du Sud en 1763. Il est d'ailleurs étonnant que la complainte ou un récit équivalent n'ait jamais été recueillis chez les descendants d'Acadiens établis ici et là le long des côtes étatsuniennes et en Louisiane.

\section{Des éléments intrigants et hors récit}

Pour ma part j'opterais pour la deuxième ou la troisième solution suggérant toutes deux un apport français direct, à cause de certains éléments, plutôt mystérieux à première vue en ce qu'ils apparaissent non pas dans la légende elle-même, mais dans l'aventure authentique de la "Colonie perdue ", à tel point que, si elle n'était pas relatée partout dans les annales historiques des États-Unis, on pourrait presque douter de son authenticité. Ainsi le nom même de la fille-biche, Virginia Dare, où Virginia signifie en tout premier lieu « virgin », (= vierge, donc déjà blanche...), et la ressemblance avec « Virginia Deer », soit « cerf de Virginie » ne peut que sauter aux yeux : on sait qu'en ancien anglais, le mot « deer», selon les époques, s'est écrit ou prononcé «der », « deor » ou « dear ${ }^{38}$, ou encore « deere » ou « deare » dans l'ancienne relation de voyage de Thomas Hariot ${ }^{39}$, et que le mot « dare » lui-même, qui signifie « défi, coup d'audace », a été anciennement prononcé " dar, der, dear, dearr ${ }^{40}$. De par son seul nom, "Virginia Dare » était donc déjà "Blanche Biche » dès son baptême. Cerfs et biches étaient d'ailleurs

37. Extrait de : L'époque coloniale 1, www3.sympatico.ca/gildore/colonialel.html

38. WNWDAL, op. cit.

39 Thomas Harriot. op. cit.

40. WNWDAL, op. cit. 
extrêmement abondants dans les parages de Roanoke et sur les côtes de Virginie : les Amérindiens de l'endroit étaient tous vêtus de peaux de cerfs, mangeaient du cerf, et les cerfs eux-mêmes venaient effrontément se nourrir à même les provisions des autochtones et des colons, comme font souvent les ours qui vivent à proximité des humains.

Les autres éléments, moins convaincants, sont néanmoins à relever dans le contexte qui nous concerne. Ainsi le nom du vaisseau commandé par le capitaine White, soit «The Lion » : on sait que le symbole du Lion est important en Angleterre, et figure même avec la Licorne sur son blason. Mais « Lion » ou « Lyon » est non seulement un très ancien prénom européen qui remonte aux temps Romains ${ }^{41}$, et en France le nom d'une ville importante, il est aussi celui du frère chasseur de la Blanche Biche dans la toute première version recueillie en France, dans l'Orne en Normandie, par le Dr JeanFrançois Gabriel Vaugeois avant 1840, et publiée après sa mort en $1841^{42}$. On ne rencontre le prénom "Lion » qu'une « vraie » fois en tout et pour tout dans le corpus, où le prénom majoritaire est Renaud ${ }^{43}$, et c'est dans cette version Vaugeois. Trois autres versions françaises utilisent le prénom Lion, mais deux sont des versions critiques ${ }^{44}$, et l'autre est la deuxième version publiée en France après celle de Vaugeois par le romancier Charles-Émile Souvestre, qui n'indique pas ses sources et ne localise pas sa version dont tout indique qu'elle provient de Loire-Atlantique, où l'auteur pourrait avoir substitué, au « René » des versions du Centre-Ouest de la France, ce

41. «Le prénom Lion : du Latin 'lion'. Prénom commun au temps romain, et c'est également le prénom de treize papes ; utilisé rarement aux XVIII'et XIX' siècles. C'est sans doute au début du $\mathrm{XX}^{\mathrm{c}}$ grâce à l'intérêt porté à l'astrologie que ce prénom a réapparu. • Dérivés: Lee, Léon, Léontios, Lev, Lion, Lyon.» (www.excessories.org/bonnefete/prenoms/l/leo.html).

42. Dr J. François Gabriel Vaugeois, Histoire des Antiquités de la Ville de L'aigle et de ses Environs. P. E. Brédif, Impr.-Libr., r. du Maure, L'Aigle, 1841 1843 (les deux dates sont inscrites au bas de la page titre). La Blanche Biche est mentionnée en page 402 et reproduite aux pages 584-585, dans les notes à la fin du livre. - Cote BN Imprimés [ $8^{\circ}$ Lk7.3349].

43. Renaut, Regnault, Ranaut, Renon, Renou, au gré des informateurs, et même René, allusion possible au Roi René d'Anjou, dont la substitution à Renaud dans plusieurs versions du Centre-Ouest de la France paraît aisée vu la proximité sonore des deux prénoms. $M^{\text {me }}$ Aurore Lamontellerie, qui voyait dans le Renaud de la Blanche Biche un héros solaire et l'assimilait à Apollon, attribuait au prénom René un sens de renaissance, de renouveau (BSMF, LXIX (N 69), $1^{\text {er }}$ mars 1968, « Le Mythe de la Blanche Biche ", pp. 173 à 184).

44. Un texte critique est un texte reconstruit, par l'auteur qui l'a publié, avec les meilleurs vers des versions de terrain qu'il connaissait à son époque, méthode éminemment subjective, on le reconnaîtra, qui résulte en une nouvelle version jamais effectivement recueillie sur le terrain. Jean-Baptiste Weckerlin a publié deux versions où le frère se prénomme « Lion », mais ce sont des textes critiques basés partiellement sur la version Vaugeois et partiellement sur la version Souvestre. 
«Lion » de la version normande qu'il avait dû trouver séduisant, peut-être parce qu'il lui trouvait un air de plus grande antiquitée ${ }^{45}$.

Un dernier élément moins évidemment français est le nom même du capitaine et gouverneur White, qui signifie «blanc». Et White était capitaine du vaisseau baptisé « Le Lion ». Ce nom est bien sûr extrêmement commun chez les anglophones, aussi commun que le nom Leblanc en français, mais il faut reconnaître qu'ici ce nom est associé à la formation d'une légende où la blancheur est de première importance. À ce nom on doit adjoindre celui de la mère du bébé, fille du Gouverneur White, épouse d'Ananias Dare, qui se nommait White. Son prénom est parfois orthographié Ellinor ou Elyonor, d'où se dégage un fonds sonore bien proche du mot français « élan », donc d'un cervidé associé à la couleur blanche : « élan blanc ». Qu'on s'entende bien, il n'est question ici que de proximités sonores et qui plus est, dans deux langues, et en aucun cas de certitudes étymologiques ${ }^{46}$.

Malgré l'évidente faiblesse argumentaire de ces éléments, comment se surprendre que dans un tel environnement physique, sonore et onomastique, un très ancien récit européen de chasse à la biche blanche ait pu prendre racine et s'incruster dans les traditions amérindiennes de l'endroit, qui comportaient probablement depuis longtemps déjà des légendes de chasse aux animaux blancs?

\section{Pour conclure}

Peut-on vraiment conclure après un exposé de cette sorte? Plusieurs informations supplémentaires seraient nécessaires, à commencer par la date où la légende de Virginia Dare a été recueillie pour la première fois, et par qui ; quelle nation amérindienne l'a d'abord transmise ; le nombre de variantes qu'on connaît à présent de ce récit; quels spécialistes l'ont étudié et comparé. Aurions-nous là un soupçon de preuve que certains membres de la « Colonie perdue » auraient survécu en allant vivre avec les Amérindiens de la région

45. À de minimes différences près, la version Souvestre est la même que celle du manuscrit Guéraud (Loire-Atlantique, c1856), oủ le frère se prénomme René, version publiée beaucoup plus tard avec des remaniements par l'Abbé Abel Soreau, en 1901. D'après mes recherches il est très probable que des membres de la même famille Praulx de la Nicollière aient communiqué séparément cette version à CharlesÉmile Souvestre, Armand Guéraud et Abel Soreau.

46. On s'intéressera cependant aux informations fournies par le WNWDAL (op. cit.) sur le mot Élan, qui se dit « Elk » en anglais, et désigne bien sûr l'orignal : [Moyen anglais, évolution irrégulière (avec $-k$ ), dérivé de l'Ancien anglais eolh, proche du Grec elch, du vieux norrois elgr, dérivé de la base germanique *alchis, dérivée d'une base indo-européenne *el-, pour stag (cerf) ou hart (cerf âgé de plus de 5 ans), d'où le gallois elain, pour biche, le lithuanien élnis, le Grec élaphos (? agneau ?]. - On se reportera également à Douglas Q. Adams (University of Idaho), «Designations of the Cervidæ in ProtoIndo-European » in Journal of Indo-European Studies, vol. 13, n 3 \& 4, Fall/Winter 1985, pp. 269-281, où l'auteur expose sa propre tentative de reconstruire une racine indo-européenne pour désigner les cervidés basée sur trois lignées de mots indo-européens dont l'une comporte une dizaine de mots à fond sonore *elEen. 
et se seraient transmis la légende entre eux d'abord, avant que commence le processus de métissage, lequel en aurait amorcé l'adaptation amérindienne?

Quoi qu'il en soit, sa présence chez les Amérindiens de Caroline du Nord et son lien possible et même fort plausible avec un récit européen, probablement français, suggère une fois de plus que des traces de nos propres traditions pourraient être retrouvées ailleurs dans les traditions amérindiennes tel, dans les légendes Mic-Macs, ce demi-dieu Glooskap plus grand que nature dont un chercheur a suggéré qu'il pourrait être un avatar du géant gallique Gargantua ${ }^{47}$. D'où l'intérêt pour les chercheurs francophones et anglophones d'être bien documentés autant sur leurs propres traditions orales que sur celles des peuples autochtones avec lesquels leurs cultures sont en interaction depuis les tout débuts des colonies européennes en Amérique.

\section{Court transit d'une version de Blanche Biche chez les Franco-Américains}

Le second volet de ce travail s'attachera à une version de Blanche Biche recueillie en 1968 dans le Bas-Saint-Laurent et qui, pour différentes raisons qu'on discutera plus loin, n'aurait pas dû se trouver à cet endroit. C'est la version qui est reproduite, en pièces détachées si on peut dire, en première partie. Remettons-la d'abord toute seule « sur le métier " pour en examiner les particularités, en relevant ses motifs principaux dans la colonne de gauche afin d'en commenter quelques-uns ensuite :

\begin{tabular}{|c|c|c|}
\hline & $\begin{array}{l}\text { Dialogue mère-fille } \\
\text { Prénom de la jeune fille, } \\
\text { Marguerite } \\
\text { Refrain : isotopie du bois }\end{array}$ & $\begin{array}{l}1 \\
\text { C'est la fille d'un roi qui s'appell' Marguerite } \\
\text { Elle est dedans un coin qui pleure, qui soupir'(e) } \\
\text { R. Et allez, allez dans le bois, dans le bois, ma mie } \\
\text { 2. Elle est dedans.../ Sa mère lui demande : } \\
\quad \text { - Qu'avez-vous Marguerit(e)? }\end{array}$ \\
\hline $\begin{array}{l}\text { 4a. } \\
\text { 4b. } \\
\text { 4c. } \\
\text { 4d. }\end{array}$ & $\begin{array}{l}\text { Bain du bébé nouveau-né, } \\
\text { bassin doré, } \\
\text { don ou sort jeté par des fées } \\
\text { irritées, } \\
\text { métamorphose nocturne } \\
\text { quotidienne }\end{array}$ & $\begin{array}{l}\text { 3. Sa mère lui.../_-Vous souv'nez-vous ma } \\
\text { mèr' le jour où je suis né(e) ? } \boldsymbol{R} \text {. } \\
\text { 4. Vous souv'nez-vous.../Vous m'avez fait } \\
\text { laver dans un bassin doré } \boldsymbol{R} \text {. } \\
\text { 5. Vous m'avez fait laver.../Vous avez fait } \\
\text { ch'ter l'eau dans le jardin des fé(es) } \boldsymbol{R} \text {. } \\
\text { 6. Vous avez fait ch'ter.../Les fées m'ont } \\
\text { donné un don, le jour où je suis fill(e) } \boldsymbol{R} \text {. } \\
\text { 7. Les fées m'ont donné.../ / Le jour où je suis } \\
\text { fille. et la nuit Blanche Biche } R \text {. }\end{array}$ \\
\hline
\end{tabular}

47. Christian David, "Le Demi-dieu Glooskap des indiens de l'ancienne Acadie française : un avatar du géant gallique ? ", dans La Lettre d'Île-de-France du Groupe Île-de-France de mythologie française, rubrique « Mythologie française hors de France », avril 2000, Nouvelle Série n 32, pp. 2-3. 
5a. Chasse (à courre) de seigneurs et de princes,

5b. Absence de chiens

2b. Prénom du frère, Gérard

6a. Médiation de la mère demandée par la fille,

6b. incrédulité du frère

7a. Mise à mort de la fille-biche

7b. avec une arme à feu

\section{Apprêts du repas (banquet)} cannibale

9a. La fille-biche morte et cuisinée révèle son identité féminine, 9b. décrit son corps morcelé

10a. Dénouement simple : Suicide du frère

$10 \mathrm{~b}$. avec son mousquet (ou plutôt son couteau)

10c. Là où il a tué sa sœur

11. Mélodie, air de chasse à courre
8. Le jour où je suis.../ La chasse est après moi, les princes et les seigneurs $\boldsymbol{R}$.

9. La chasse est après.../Et mon frère Gérard qui-en vaut autant que quatre $\boldsymbol{R}$.

10. Et mon frère Gérard.../Allez lui dir', ma mère, allez lui dir' qu'il cesse $\boldsymbol{R}$.

11. Allez lui dir',.../ - Cesse, cesse Gérard, c'est ta sœur Marguerite $\boldsymbol{R}$.

12. - Cesse, cesse Gérard,.../ - Ma mèr' je n'comprends rien de ce que vous me dites $\boldsymbol{R}$.

13. - Ma mèr', je n'comprends.../Gérard prit son mousquet par trois coups, il tir(e) R.

14. Gérard prit son.../ Et au troisième coup, la Blanche Biche est prise $\boldsymbol{R}$.

15. Et au troisième coup.../ - Allez, allez ma mère porter à la cuisine $\boldsymbol{R}$.

16. - Allez, allez ma mère.../ Et dites aux cuisinières qu'ell's la fassent bien cuire $\boldsymbol{R}$.

17. Et dites aux cuisinières.../Venez, venez Marguerite goûter à la cuisine $\boldsymbol{R}$.

18. Venez, venez.../Mon frèr' j'suis à la table, la première mise $\boldsymbol{R}$.

19. Mon frèr' j'suis.../ Mon corps est dans un plat, au milieu de la table $\boldsymbol{R}$.

20. Mon corps est.../ Mon cour à la cuisine, cloué à un' cheville $\boldsymbol{R}$.

21. Mon cœur à la .../ Gérard prit son mousquet (son couteau), à la forêt s'enfuit $\boldsymbol{R}$.

22. Gérard prit son.../ On retrouva son corps où tomba Blanche Bich(e)

$\boldsymbol{R}$. Et allez, allez dans le bois, dans le bois, ma mie

Autant il a fallu « ratisser large » pour regrouper des documents sur Virginia Dare en procédant par navigation sur la toile, autant le jeu de piste visant à retracer l'itinéraire de la version Lavoie/Pouliot de la Blanche Biche a dû se baser, par la force des choses, sur un nombre limité de documents et d'attestations.

Une publication plus élaborée sur cette complainte étant prévue, qui comportera une discussion de l'ensemble des motifs, ne seront commentés 


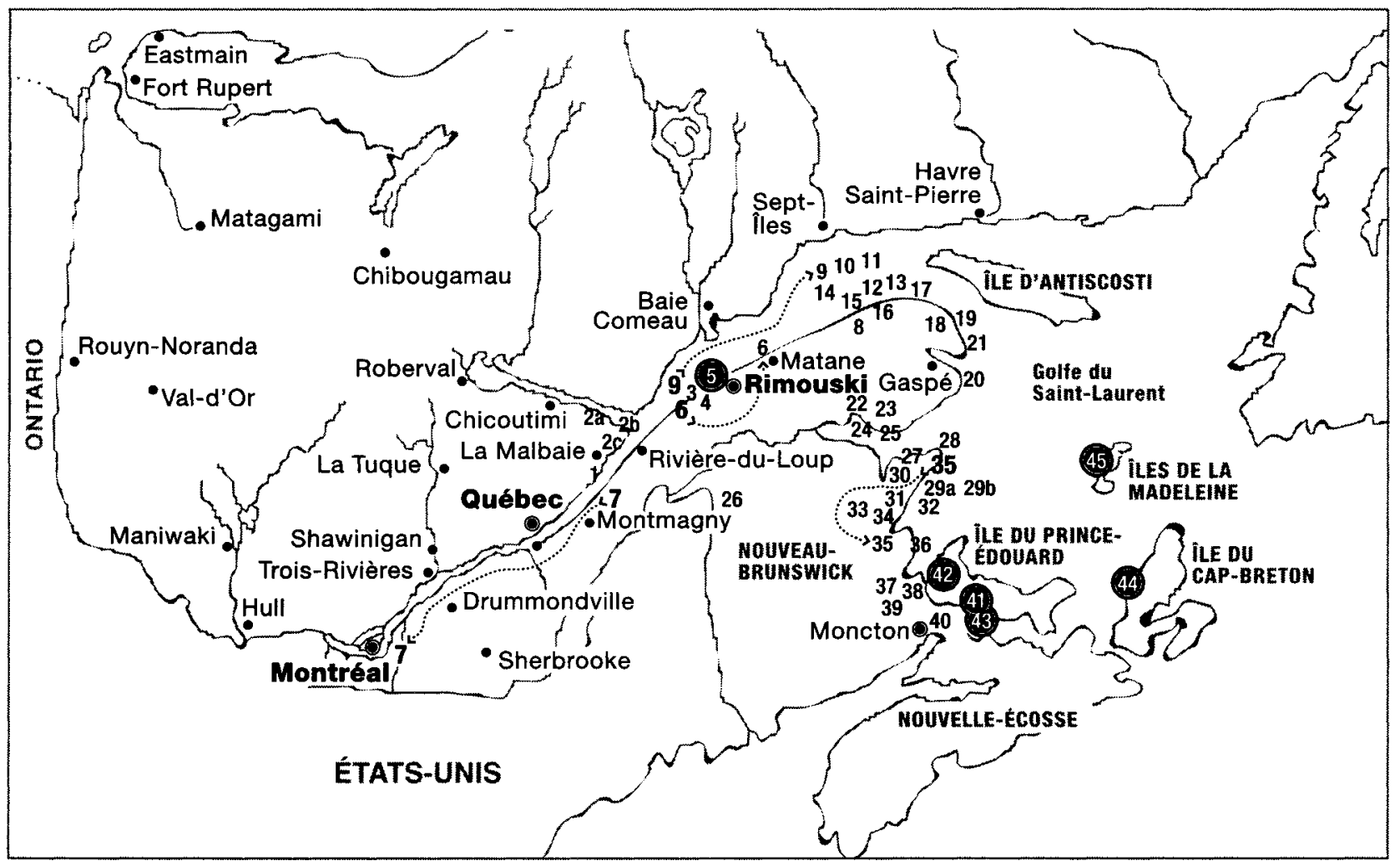

Blanche Biche - Localisation des versions au Québec et en Acadie • Versions à refrains : dans les cercles noirs - Pour certaines versions, on connaît une provenance plus ancienne que l'endroit où elle a été recueillie : ces déplacements sont tracés en pointillés, du lieu de départ connu aux lieux de collecte. 
ici que les motifs pouvant étayer l'argument de ce travail, soit le refrain, les prénoms des héros, le dénouement, et la mélodie.

Voici d'abord une carte, localisant géographiquement les 45 versions du Canada francophone recueillies sur le terrain (25 versions au Québec, 20 en Acadie). Carte un peu sommaire je l'admets, pour cause de manque d'espace et de « grappes » de versions recueillies aux mêmes endroits (Gaspésie-Nord, Port-Daniel ; Nord-Est du Nouveau-Brunswick). Mais elle permet de comprendre d'un coup d'œil la répartition géographique canadienne de la Blanche Biche, et la position particulière des versions à refrain (chiffres blancs encerclés de noir).

\section{Commentaire des motifs}

1. Refrain - Isotopie du bois : Sur l'ensemble du corpus, douze versions comportent des refrains, soit six versions canadiennes, cinq versions scandinaves dont une version critique, et une version française. Neuf de ces refrains manifestent l'isotopie du bois ou de la forêt, incluant la version critique. Mais au Canada, la présente version à refrain est la seule qui ait été recueillie au Québec. Les cinq autres proviennent d'Acadie, plus précisément des provinces maritimes îliennes.

Voici les énoncés de ces douze refrains, placés l'un sous l'autre pour fins de comparaison :

1. Suède 1570, v.o.: [...] Dans la forêt [...] qui porte l'or sous ses flancs ${ }^{48}$

2. Suède 1670 , v.o.: $\quad[\ldots]$ Voyez les animaux dans l'île [...] Comme ils courent joyeusement $t^{49}$

3. Danemark 1844 , v.o. : [...] Qui portait de l'or rouge [...]

4. Danemark 1873, v.o. : Qui portait l'or au-dessus de sa tête ${ }^{50}$

[...] Dans la forêt [...] Qui porte l'or au-dessus de sa tête

48. S1570Aa : [...] I skokenn! [...] Som gull bärer vnder sine boger (Harald Oluffson Visbok 1570, Svenst Visarkiv, Stockholm : v.o. recueillie dans le district de l'Uppland). op. cit.)

49.S1670Aa: [...] Se djuren under ô [...] Sa lustelig sa rinna de! (SMB, op. cit., et A.I. Arwidsson,

50. D1844a: [...] Som rode Guld bad [...] Som Guldet bar over sit Hoved (Svent Grundtvig, “ Jomfruen I Hindeham " Danske Gamle Folkeviser II (DGF) 1854-1856. Ballade n 58, Version A, 50 couplets. Informatrices : Margrethe Nielsdatter>Elisa Galskiøt, de Slaglille -v.o. probablement apprise d'une feuille volante).

51. D1873a : [...] I Skoven [...] Som Guldet bar over sine Bover (Evald Tang Kristensen, Jyske Folkeviser, Viborg, trykt hos F.V. Backhause, 1889, $\mathrm{n}^{\circ} 7$, pp. 14-15. Informatrice : Ane Margrete Eskildsdatter v.o., de Torning). 
5. Danemark/Suède, v.c. 1899 :

[...] Dans la forêt $[\ldots]$ Qui porte l'or sous ses flancs ${ }^{52}$

6. Île-du-Prince-Édouard, 1926, v.o. : Dans les bois, dans les bois, ma mie $e^{53}$

7. Île-du-Prince-Édouard, 1958, v.o. :

Qu'é dans le bois / Dans le bois ma mie ${ }^{54}$

8. Île-du-Prince-Édouard, 1975, v.o. : Dans le bois, dans le bois ma mie $e^{55}$

9. Île-du-Cap-Breton 1946, v.o. : Dans les bois, dans les bois, ma mie, dans les bois ${ }^{56}$

10. Îles-de-la-Madeleine, 1978, v.o. :

11.Québec, Bas-St-Laurent, v.o. 1968 :

12. France, Poitou, $1884-85$, v.o. :
On les voit, on les voit, ma mie s7 $^{57}$

Et allez, allez dans le bois /

Dans le bois, ma mie ${ }^{58}$

O mon Dieu, donnez-moi

ce que mon cour désir ${ }^{59}$

En consultant cette liste, on constate que l'unique refrain français $\left(n^{\circ} 12\right)$, à caractère d'invocation religieuse, est très atypique, probablement d'insertion tardive dans la chaîne de transmission de cette complainte, et ne peut vraiment servir notre propos. On est aussi tenté d'imaginer une transmission de source scandinave sur les côtes canadiennes : le refrain suédois $\mathrm{n}^{\circ} 2$ (1670) ne comporte-t-il pas le verbe " voir » ("Voyez les animaux...etc $»)$, qu'on retrouve dans le refrain $n^{\circ} 10$ des Iles-de-la-Madeleine ("On les voit ») ? De plus, deux refrains scandinaves comportent l'expression " dans la forêt » : celui de la version suédoise recueillie en 1570 , soit 34 ans avant l'établissement d'une colonie française en Acadie ( $\left.\mathrm{n}^{\circ} 1\right)$, et celui de la version danoise datant de $1873\left(\mathrm{n}^{\circ} 4\right)$; de leur côté les canadiens disent six fois " dans les bois » si on inclus la même version $n^{\circ} 10$, en supposant cette fois que l'informatrice des Iles-de-la-Madeleine ait transformé le mot « bois » en « voit », ce qui serait plausible vu la proximité sonore des lettres «b» et « $\mathrm{v} »-$ ainsi va la langue d'ailleurs et ses fluctuations de sons, comme pour le «b-v » espagnol...

52.D1899 : [...] I skove [...] Som guldet bcer under Bove (Axel Olrik Danske Folkeviser, Kobenhavn, Gyldendalske Boghandels forlag (F. Hegel \& So), Fr. Bagges Bogtrykkeri, 1899, nº 12, pp. 44-45. (version critique, bases Suède et Danemark).

53 C1926A : Coll. Abbé P.Paul Arsenault, CCÉCT, Hull, Qc, mél. n 51, Mont-Carmel, ÎPÉ, v.o.

54 C1958Aa : coll. Lacourcière, AF enr. 3666, 3-1, 1958 / Marguerite Gallant (Mme Philippe Arsenault), v.o. St-Chrysostôme, Baie Egmont, ÎPÉ.

55 C1975 : coll. G. Arsenault CÉA Moncton, Bob. 63, enr. 1982, Marianne Gallant, v.o., StÉdouard, ÎPÉ.

56. C1946Db : Coll. G. Massignon Trésors de la chanson populaire française, thèse de doctorat 1962, réédition, revue et corrigé par Georges Delarue BNF, Paris, 1994, BB, pp. 34 -39. / M. Germain Chiasson, v.o. St-Joseph-du-Moine, Île-du-Cap-Breton, N.-É.

57. C1978Ba : coll. Suzy Leblanc, AF enr. 149 / Mme Adeline Lapierre, v.o. Fatima, Îles-de-laMadeleine, Qc.

58. C1968a, b, c : coll. Y. Rhéaume 1968, coll. Reeves/DeGrosbois 1993>1998 / Famille Lavoie/ Pouliot, v.o.

59. F1884-85 : Eugène Rolland, Revue Mélusine II, Paris, 1884-1885, p. 369, en note 1, v.o. du Poitou, France. 
L'isotopie du bois ou de la forêt est tout à fait appropriée dans cette complainte de chasse à la biche, d'où la probabilité que ces refrains soient partie intégrante du récit plutôt que « rapportés », comme beaucoup de refrains dans les chansons de danses ou de voyageurs par exemple, qui n'ont rien à voir avec les chansons et dont le but est souvent de les allonger humoristiquement ou rythmiquement pour faire durer la danse ou le rire. Au contraire, ici ce motif du bois ou de la forêt où la chasse fratricide se déroule, le plus souvent de nuit, vient renforcer la tension dramatique du récit. Il en a déjà été plus longuement discuté dans la thèse de Thierry Charnay sur la Blanche Biche ${ }^{60}$, à laquelle on pourra se reporter, le propos immédiat de ce travail étant de démontrer que la version à refrain de la famille Lavoie/Pouliot de Rimouski est " hors place " au Québec, où les 24 autres versions québécoises n'ont pas de refrain et se chantent toutes sur une autre ligne mélodique, constante d'une version à l'autre et plus ou moins élaborée, que j'ai baptisée « timbre canadien majoritaire », "TCM» pour faire plus court, les autres mélodies étant alors appelées «timbre canadien différent avec refrain» ou «TCdR ».

- Le prénom du frère, Gérard : Le prénom habituel et majoritaire du frère dans cette complainte est Renaud, comme il a été dit. Sur l'ensemble des 84 versions du corpus, incluant les onze versions critiques, il apparaît 40 fois, et Marguerite 63 fois. Dans certaines versions, les héros n'ont pas de nom et sont appelés simplement le «frère aîné » ou « la sœur ». Pour la fillebiche on compte sept autres prénoms n'apparaissant qu'une ou deux fois chacun : Catherine deux fois, Laurencine, Angélique, Argentine, Louise deux fois, Hélène deux fois, Little Kirsten, et dans des contes, Magdeleine et... huit avec Virginia Dare peut-être ? Les substitutions de prénoms concernent plus souvent Renaud le frère, mais semblent parfois liées soit à de la mythologie chrétienne, soit à des événements historiques qui ont frappé l'imagination de la population : on compte trois Biron en Ille-et-Vilaine ${ }^{61}$, Julien une fois en France et trois fois au Canada francophone; Judas trois fois au Québec, Jean une fois en France, ces deux derniers prénoms ayant peut-être été substitués suite aux mêmes tragiques événements historiques que le prénom Julien ${ }^{62}$. Plus énigmatiques sont, au Canada, les autres prénoms

60. Thierry Charnay, op. cit., pp. 200-201

61. Dans les trois versions d'Ille-et-Vilaine publiées par Jean-Jacques Ampère en 1854 et Lucien Decombe en 1884. Pour avoir comploté contre le roi Henri IV, le Maréchal Biron a été décapité à Paris en 1602 , un an avant les amants incestueux Julien et Marguerite de Ravalet, devant la même foule de badauds certainement. Son nom est passé dans plusieurs chansons, et deux complaintes rapportent cette histoire de deux points de vue différents, l'un partisan et l'autre accusateur.

62. Supra. Le père et le frère aîné de Julien et Marguerite de Ravalet se prénommaient tous les deux Jean. Et Julien de Ravalet, au cours de son procès, disait vouloir être damné comme Judas si le fait dont on l'accusait était véritable. 
du frère : Guillaume (prononcé Dillaume) trois fois, Gérard quatre fois, et une seule fois Louis et Honoré. On joindra à Honoré le fameux Lion de la version Vaugeois de Normandie ${ }^{63}$ pour leur possibilité de lien avec les récits du martyre de sainte Marguerite.

La place essentielle des prénoms Marguerite et Renaud dans cette complainte a été discutée dans un autre travail, encore inédit ${ }^{64}$, mais comment et pourquoi le prénom Gérard y a-t-il été inséré ? En 1992, au début de mes enquêtes dans la famille Lavoie/Pouliot (cinq filles, un garçon), à Rimouski, je ne connaissais qu'une autre version où le frère se prénommait Gérard. Apprenant que le seul garçon de cette famille se prénommait Gérard, j'ai d'abord assumé que la maman, Louise-Anna Lavoie, avait changé le prénom du héros pour amuser son fils, comme elle l'avait fait dans une autre chanson enfantine, " La poulette grise » (« ... pour Gérard qui va faire dodo "). Mais en compulsant à nouveau mes fiches de versions, auxquelles d'autres se sont ajoutées depuis 1992, j'ai compté quatre Gérard, dans deux versions du Bas-Saint-Laurent, une de l'Île-du-Prince-Édouard, et la dernière du Centre-Est du Nouveau-Brunswick, face à la pointe nord de l'île-du-PrinceÉdouard, recueillies dans des familles a priori non apparentées. Voici une nouvelle carte de localisation où cette fois les versions « à Gérard(s)» sont à fond blanc entouré de noir, suivie d'une liste détaillée de ces quatre versions :

63. «Une légende de Provence (Guide de la Provence mystérieuse, Tchou, 1976, pp. 243-244), issue des Îles de Lérins, raconte l'Histoire de sainte Marguerite et de son frère Honorat qu'elle aimait tendrement et dont elle ne voulait pas se séparer au point de faire des miracles pour le voir ». Cité sans plus d'analyse par Thierry Charnay, op. cit. p. 198. Un lien existe sûrement entre les récits du martyre de sainte Marguerite et la complainte de la Blanche Biche, ne fut-ce que par l'importation de motifs et de vers d'un récit à l'autre. Ainsi Olibrius, le bourreau de sainte Marguerite, est plusieurs fois assimilé à un chien et à un lion... Un vers du « Cantique de sainte Marguerite» «Ainsi que d'une fontaine / S'en va le sang coulant » utilise des termes rappelant ceux d'une version de Loire-Atlantique de la complainte : "Dans le sang que ma mère avait mis dans nos veines / J'ai laissé boire mes chiens comme à l'eau des fontaines "; ailleurs, les vers « Mon âme assurément ira en paradis $[. .$.$] / A jamais damné vous serez "$ semblent être passés tout droit du cantique aux dénouements moralisants des versions de Blanche Biche du Canada et du Centre-Ouest de la France (Comte Jules de Douhet, « Légende de sainte Marguerite [en vers], fournie par la Bibliothèque bleue », dans Dictionnaire des légendes du christianisme, publié par l'abbé Migne, Turnholti, 1989, pp. 824-829).

64. F. B. Reeves, «Une biche blanche comme le lait - A Milk-White Hind», communication inédite présentée au Congrès de l'ACEF à Sudbury en mai 2002, sous le titre transitoire « La Blanche Biche, une affaire de prénoms?" 


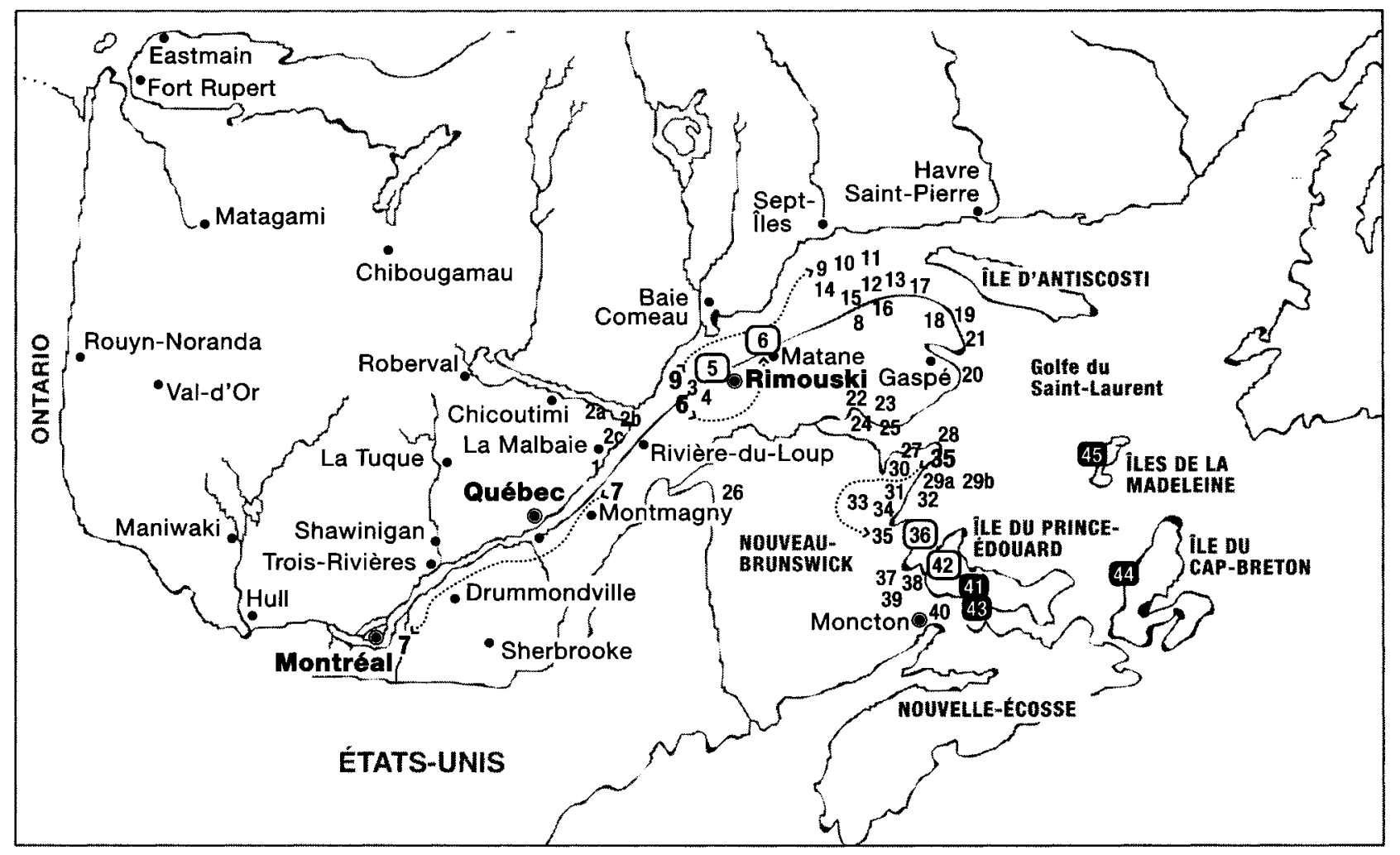

Blanche Biche, localisation des versions à prénom Gérard 
$\mathbf{N}^{\circ}$ 5. C1968a et b, coll. Yolande Rhéaume 1968 et F. Reeves 1993 à 1998 : Famille Lavoie/Pouliot, Nazareth (Rimouski), Bas-St-Laurent. Les chanteuses, Stella Pouliot (Mme Sylvio Proulx) et sa sœur Jeanne d'Arc POULIOT (Mme Roger Lepage).

Dénouement simple : suicide du frère, "Gérard prit son mousquet (ou son couteau), à la forêt s'enfuit/On retrouva son corps où tomba Blanche Biche ».

$\mathbf{N}^{\circ}$ 6. C1977A, Coll. Père Germain Lemieux, s.j., CFOF, enr. 3660, GM2 40-42 (547-639), Ms 115,16, et coll. Turcotte/Bélanger, AF et UQAR, enr. 27 (177286). Informatrice : Adélaïde Paquet ( $M^{\text {me }}$ Victor Jean), née et élevée à Rivière Blanche, St-Ulric-de-Matane, Bas-St-Laurent. Enregistrée à Sayabec, Matapédia. Sa mère, une demoiselle Adèle Dion, de Cacouna (Bas-StLaurent), épouse de Pierre Paquet, qui lui a transmis la majorité de ses chansons, était native de Cacouna, plus bas sur la côte du Bas-Saint-Laurent.

Dénouement simple : suicide du frère, "Gérard prit son couteau à la poigne d'or fine / Trois coups s'en est donné, trois coups à la poitrine / Voilà donc Gérard mort, et sa saur Marguerite "

$\mathbf{N}^{\circ}$ 36.C1992, coll. Jean-Pierre Pichette et Robert Richard. Informatrice : $\mathbf{M}^{\mathrm{mc}}$ Séraphie Daigle-Martin, Richibouctou, N.B. La chanteuse, décédée deux ans après cet enregistrement, native de Baie-Sainte-Anne, où elle a d'ailleurs été enterrée, disait tenir cette chanson de son père.

Dénouement simple, motif du suicide du frère atténué en « mea culpa » du Confiteor : Transformation du suicide du frère en dénouement à caractère religieux ? (voir version de Mme Jean, $\mathrm{n}^{\circ} 4$ ci-dessus, qui utilise des termes presque identiques au milieu de l'énoncé : «Gerârd lui frapp' troës c(r)oups dans sa blanche poitrine / En disant :C'est d'ma faute, ah ! oui c'est par ma faute / C'est par ma très grande faute $)$.

$\mathbf{N}^{\circ}$ 42. C1958Aa, coll. Lacourcière, $\mathrm{AF}$ enr. $\mathrm{n}^{\circ}$ 3666, 3-1. Informatrice : Marguerite Gallant ( $\mathrm{M}^{\mathrm{me}}$ Philippe Arsenault), Baie Egmont, Mt-Carmel, Île-du-PrinceÉdouard. La chanteuse prononce « Girofe », mais à l'écoute on constate qu'une autre chanteuse non identifiée assiste à la séance d'enregistrement, qui aide tout au long l'informatrice à se souvenir de la chanson et en complète le dénouement : elle prononce bien « Gérard » le prénom du frère.

Dénouement à deux motifs : a) à caractère religieux : promesse d'enfer pour le frère et de paradis pour la sœur; b) Suicide du frère, "Gérard prit son épée, au cour se l'a plantée »

Ces quatre familles non apparentées étaient-elles toutes pourvues d'un père, d'un frère ou de quelque autre parent dont on aurait glissé le prénom Gérard dans les chansons de leurs répertoires familiaux ? C'est une possibilité, difficilement vérifiable, que vient cependant affaiblir un vieux conte wallon 
dont le héros, prénommé Gérard, a partie liée avec une biche blanche. En voici le résumé, publié par Jean-Loïc Le Quellec ${ }^{65}$ qui le présente en ces termes :

"Autre sorte de Blanche Biche $\sim$ où le noir et le blanc, le blanc et le rouge (rouillon) sont à nouveau liés, et où on a un Gérard lié à une biche blanche ».

À propos de la Roche aux Corneilles d'Annevoie-Rouillon (Wallonie) : "A propos de la Roche aux Corneilles d'Annevoie-Rouillon (Wallonie) : Gérard était le cadet de dix enfants. Son père eût voulu en faire un chanoine, mais il préféra la profession de trouvère. Ayant passé une semaine au château du comte de Namur, il s'en fut le long de la Meuse pour visiter les manoirs qu'il rencontrerait. Il se reposa au pied du rocher de Rouillon. Une fée lui apparut et lui proposa son amour. En partant, elle lui remit un cor. Dès qu'il soufflait dans cet instrument, une biche blanche apparaissait et le conduisait auprès de son amante. Gérard ne chanta plus et se consacra à son amour. Après un an, la fée disparut. La reine des fées lui reprocha d'avoir détourné un ménestrel de sa voie. Pour la punir, elle la transforma, ainsi que Gérard, en corneille, et elle leur donna pour domaine le rocher de Rouillon. C'est pourquoi ce roc porte le nom de Roche aux Corneilles ${ }^{66}{ }^{66}$

On a donc ici un homme prénommé Gérard, comme dans quatre versions canadiennes de la Blanche Biche; ce n'est pas un chasseur, mais un trouvère. Cet homme souffle dans un cor, comme le frère-chasseur dans d'autres versions de la complainte, et ses rencontres amoureuses avec une fée sont aussi initiées par une biche blanche, qui n'est pas sa sœur mais certainement une autre fée, sinon la même, donc un être qui influera sur son destin ( fatum »). Ici les deux amants sont punis et métamorphosés en corneilles.

J'ai discuté plus à fond ailleurs ${ }^{67}$ de cet enchevêtrement apparemment inextricable du blanc et du noir souligné par Le Quellec : dans ce conte, blancheur de la biche, qui pourrait être la fée-amoureuse elle-même prenant forme humaine au moment voulu ; noirceur des corneilles. Pour le rouge (rouillon), suggérant le sang, souvent présent à côté du noir et du blanc dans les récits traditionnels, son évidence est plus apparente dans d'autres récits que dans celui-ci et dans cette version de la complainte, qui pourraient avoir

65. Jean-Loïc Le Quellec, 1993, " Chênes et chouans » [CHCH], dans La Vendée autrement dite, Mougon, Geste, pp. 28-45.

66. Pierre Saintyves, Conte des bossus, 1936, pp. 266-267 ( $\left.{ }^{\circ} 1791\right)$. (ordre des mots changé)

67. F. B. Reeves, 2002, op. cit. 
été amputés de passages clarifiants à cet égard. Mais six versions de la Blanche Biche mentionnent spécifiquement le sang. En voici les énoncés, où aucune version canadienne ne figure ; seules deux versions scandinaves et quatre françaises comportent ce motif, qui est néanmoins implicitement présent dans toutes les versions par la simple mise à mort de la fille-biche :

1. "Là tombe le sang le long du ruisseau » (Suède, OlufFson S1570a)

2. "Herr Peder se coupa les dix doigts / Ma chère sœur, bois maintenant de cela / Quand elle but le sang de son frère / Elle redevint une bonne vierge " (Danemark, GrundTVIG, D1844a et D1874a)

3. «Jamais je ne verrai ni buisson ni épines / Qu'ils ne soient arrosés du sang de Marguerite » (France, TREBucQ F1896a);

4. «Mon sang est répandu par (ou sur) toute la cuisine » (France, Ille-et-Vilaine, Ampère/Roulin, F1853Aa ; Compaing/Pineau, Poitou, F1892a)

5. «Et là mon sang coule à grand flot, mon âme italie (= s'en est allée ? étalée ?)» (France, La Garnache, Vendée, Cahier manuscrit de Dame Georgette BLANCHARD-MORRISSON, F c1930-40a)

6. "Le bras du dépouilleur est rouge jusqu'à l'aisène » / " Dans le sang que ma mère avait mis dans nos veines / J'ai laissé boire mes chiens comme à l'eau des fontaines " (France, Loire-Atlantique, version SGS (SouvESTRE/ GUéraUd/SOREAU) F1849-51 Ia, IIa, b)

Même si l'aventure de Gérard de Rouillon diffère grandement du récit de notre complainte, elle permet de supposer aux quatre Gérard des Blanche Biche canadiennes des racines beaucoup plus anciennes que je l'imaginais au début de mes enquêtes dans la famille Lavoie/Pouliot.

Dénouement, suicide du frère : Il est remarquable que ces quatre versions à prénom Gérard comportent toutes le motif du suicide du frère reconnaissable quoique atténué en mea culpa dans la version de Mme Séraphie Martin -, motif plutôt rare puisqu'on ne le rencontre que huit fois au Canada (quatre fois au Québec, quatre fois en Acadie), une fois en Suède, et pas du tout en France. Ce motif, qui préserve le caractère tragique et fataliste de la complainte, est probablement assez ancien, à la différence du plus fréquent dénouement à caractère religieux (dix-huit occurrences en France, en Acadie et au Québec), où la sœur voue son frère aux flammes de l'enfer et se promet à elle-même le paradis, motif d'insertion sûrement plus récente dans la longue histoire de la complainte, et qui jette un blâme moralisant sur le drame fratricide du récit.

La mélodie : les mélodies des autres versions à refrain (TCdR) ont quelques affinités entre elles ou avec le timbre majoritaire canadien, mais la 
mélodie de la version Lavoie/Pouliot ne peut leur être associée. En voici le premier couplet :

Tempo : noire $=90$

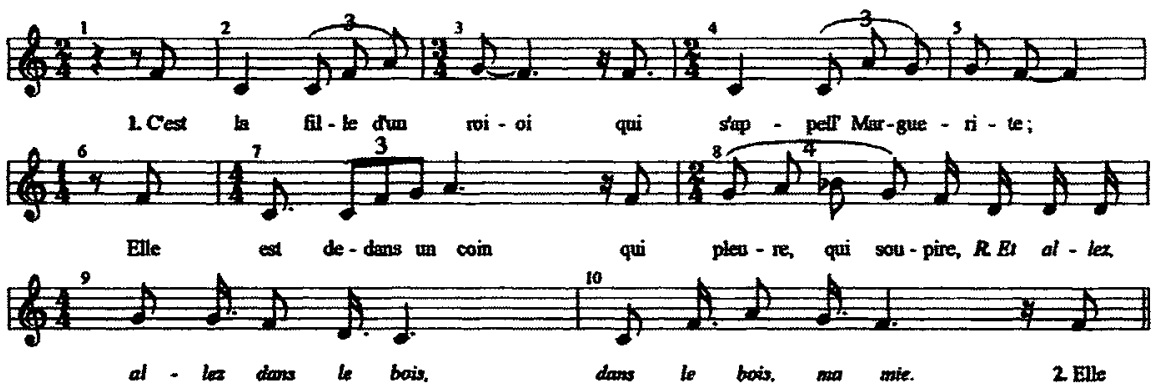

Blanche Biche, version de la famille Lavoie/Pouliot, de Nazareth, Rimouski. Mélodie du premier couplet / Notation FR.

Cette mélodie est unique dans le corpus et présente toutes les caractéristiques d'un air de chasse à courre. On ne peut pour l'instant que souligner ce fait en se questionnant sur son origine, qui pourrait éventuellement servir l'argument de ce travail.

Provenance de la version Lavoie/Pouliot : Les questions que je me posais sur cette version m'ont amenée à enquêter plusieurs fois auprès de cette famille ${ }^{68}$, qui s'est toujours montrée très accueillante, généreuse et coopérative. Deux des filles, Stella Pouliot ( $\mathrm{M}^{\mathrm{me}}$ Sylvio Proulx) et Jeanne d'Arc Pouliot ( $\mathrm{M}^{\text {me }}$ Roger Lepage) ont pu me chanter la chanson en entier, les autres sœurs ne se souvenant que de bribes. $\mathrm{M}^{\mathrm{me}}$ Proulx avait déjà été enregistrée par Yolande Rhéaume en 1968, et avait à ce moment soigneusement revu « sa copie ", effectuant même quelques rectifications qui lui semblaient plus logiques. $\mathrm{M}^{\mathrm{me}}$ Lepage de son côté nous a chanté la chanson presque au pied levé, puisqu'elle est arrivée chez sa sœur $\mathrm{M}^{\mathrm{me}}$ Proulx pendant une séance d'enregistrement, et s'est gentiment laissé enregistrer aussi. D'où de légères différences avec la version de sa sœur, dont le couteau comme instrument du suicide du frère ("M'semble que maman disait 'son couteau'... »), $\mathrm{M}^{\mathrm{me}}$ Proulx admettant l'avoir corrigé en mousquet, qui lui semblait plus logique puisque c'était l'arme utilisée juste avant par le frère pour tuer la biche. Elle a plus tard reconnu en riant qu'il devait être très difficile de se suicider avec un mousquet, vu la longueur de l'arme !

68. Enquêtes effectuées en 1993, 1997, 1998 (tournage vidéo, coll. Reeves/De Grosbois). 
Lors de mes enquêtes je leur ai plusieurs fois demandé si leur famille avait de la parenté acadienne ${ }^{69}$. La réponse a toujours été négative. Quand j'ai mentionné à des chercheurs connaissant mieux que moi l'Acadie ${ }^{70}$ cette version à refrain qui n'aurait pas dû se trouver au Québec, ils m'ont appris qu'au début du $\mathrm{XX}^{\mathrm{e}}$ siècle, plusieurs familles acadiennes de Rustico, sur l'Île-du-Prince-Édouard, avaient quitté leur île pour venir s'installer au Québec dans la vallée de la Matapédia. Cette explication aurait pu être satisfaisante, puisque Émile Pouliot, père des Lavoie/Pouliot était fils de Victor Pouliot, qui avait été maître-chantre pendant 32 ans dans la Matapédia, à l'église d'Amqui, village où il résidait.

Toutefois les enfants d'Émile Pouliot ne tiennent pas leur Blanche Biche de leur père, mais de leur mère, Louise-Anna Lavoie, dont le périple familial a été extrêmement différent. En effet, l'année de sa naissance, en 1888, LouiseAnna quitta Rimouski avec sa famille qui émigrait aux États-Unis et devait s'installer à Fall-River, dans le Massachusetts. Elle ne revint pour la première fois à Rimouski que trente ans plus tard, en 1918, pour une courte visite à sa parenté, mais sa rencontre décisive avec Émile Pouliot changea ses projets : elle épousa Émile peu après, le couple s'établit à Rimouski, et leurs six enfants naquirent à intervalles réguliers dans les années qui suivirent. Elle ne revit Fall-River que très longtemps après, avec sa fille Stella et son gendre M. Sylvio Proulx qui l'y conduisirent en voiture.

Louise-Anna avait rapporté avec elle un imposant bagage de chansons traditionnelles, certainement apprises à Fall-River dans les milieux francoaméricains. D'après ses filles elle chantait tout le temps, de sorte qu'elles disent avoir appris sa version de la Blanche Biche " pas mal proche du berceau $»$.

Sachant qu'un répertoire de chansons traditionnelles se construit pendant les vingt ou trente premières années d'une vie, il paraît évident que Louise-Anna avait appris sa Blanche Biche à Fall-River, aux États-Unis. Mais si cette chanson a eu cours à cet endroit au tournant du siècle, elle ne semble pas y être restée, du moins pas à ma connaissance. Je n'ai pas eu la possibilité de consulter les collections franco-américaines de chansons traditionnelles, et j'ignore si elles ont été complètement dépouillées, mais je sais qu'entre

69. On pourrait se questionner aussi sur les éventuelles origines acadiennes de l'autre famille québécoise à version " Gérard » que je n'ai pas eu la possibilité de visiter, celle de $\mathrm{M}^{\text {me }}$ Victor Jean (Adélaïde Paquet), de St-Ulric-de-Matane, qui tenait sa version de sa mère native de Cacouna dans le Bas-Saint-Laurent.

70. Ronald Labelle, du CÉA de Moncton, N.-Br. ; Donald Deschênes, SQE, Québec ; Jean-Pierre Pichette, Univ. de Sudbury, Ont. (conversation à quatre) 
autres la collection d'Adélard Lambert ${ }^{71}$, qui a vécu longtemps à Manchester, non loin de Fall-River, au début du $\mathrm{XX}^{\mathrm{e}}$ siècle, ne contient pas de Blanche Biche.

Toujours intriguée par ce problème, à l'occasion d'une nouvelle enquête à Rimouski auprès des sœurs Pouliot, en $1997^{72}$, je demandai la permission de consulter leur dossier généalogique familial, préparé par leur frère Gérard. Après en avoir compulsé quelques pages en cherchant d'une ligne à l'autre des localités acadiennes, je trouvai le nom de Charlottetown, principale ville de l'Île-du-Prince-Édouard. Hélas, la personne qui avait vécu là était une religieuse, sœur Saint-Paschal, donc une personne qui n'avait pas eu d'enfants. Je me préparais à continuer mon inspection du classeur lorsque $\mathrm{M}^{\mathrm{mc}}$ Proulx m'apporta la rubrique nécrologique de cette religieuse, qui était une grandtante de son père. Voici en résumé ce qu'on pouvait y apprendre :

Sœur Saint-Paschal, des sœurs de la Charité, ou sœurs Grises de Québec, née Henriette Pouliot en 1839 à Saint-Germain-de-Rimouski, décédée à 84 ans le 9 mars 1923, était l'arrière-grand-tante paternelle des Lavoie/Pouliot. Cette religieuse a été très " mobile " tout au long de sa vie religieuse, séjournant plus ou moins longtemps à divers endroits selon les ordres de sa communauté, qui l'envoyait ici diriger un noviciat, ailleurs un hôpital comme elle l'a fait pendant quatre ans à Charlottetown à l'île-du-Prince-Édouard entre 1884 et 1888, ailleurs encore un orphelinat comme elle l'a fait en 1890, justement à Fall-River aux États-Unis, où elle est restée assez courtement semble-t-il, ayant été vite rappelée au Québec par ses supérieures pour mener à bien d'autres œuvres de fondation.

Ces pérégrinations d'une religieuse au $\mathrm{XIX}^{\mathrm{e}}$ siècle nous proposeraientelles un début d'explication à cette Blanche Biche qui aurait dû se trouver du côté de l'île-du-Prince-Édouard plutôt qu'à Rimouski au Québec ? Il y a en tout cas correspondance de dates et de lieux :

Louise-Anna Lavoie, qui était encore un bébé, était déjà à Fall-River depuis 1888 quand sœur Saint-Paschal est venue y fonder un orphelinat en 1890. Cette religieuse aurait pu avoir appris une Blanche Biche avec d'autres chansons à Charlottetown, quand elle y a dirigé un hôpital pendant quatre ans, et transporter cette chanson avec elle ici et là au gré de ses mandats de fondations. Étant originaire de Rimouski, il semble normal qu'elle ait fréquenté la communauté franco-américaine de Fall-River venue de Rimouski également. Que ces gens aient chanté ensemble et qu'elle leur ait transmis quelques chansons apprises ailleurs, dont la Blanche Biche, semble éminem-

71. Les collections d'Adélard Lambert sont déposées à différents endroits, dont les locaux de l'Association Canado-Américaine à Manchester aux États-Unis. Sa collection de chansons est conservée au CCÉCT de Hull.

72. Enquête Reeves/G.Breton dans la famille Lavoie/Pouliot en 1997. 
ment plausible également. Peut-être n'est-elle pas restée assez longtemps pour la transmettre directement à Louise-Anna Lavoie, qui n'avait alors que trois ou quatre ans, mais la chanson a pu séjourner un certain temps à FallRiver après le départ de la religieuse, assez longtemps pour que Louise-Anna se l'approprie et la rapporte trente ans plus tard en 1923 à Rimouski, où la chanson se chante encore chez ses descendants à qui elle l'a transmise alors que, selon leurs dires, ils étaient encore " pas mal proche du berceau ".

Sœur Saint-Paschal aurait pu aussi transmettre la complainte à ses collègues religieuses de Rimouski, dont certaines auraient été envoyées à Fall-River ensuite pour s'occuper de l'orphelinat, établissement que LouiseAnna Lavoie aurait pu fréquenter à un moment ou un autre, juste assez de temps pour apprendre la complainte.

\section{Pour conclure}

Parle-t-on encore français à Fall-River en 2004 ? Y chante-t-on encore en français les vieilles chansons traditionnelles? Si la Blanche Biche a disparu du répertoire après avoir eu cours parmi quelques familles franco-américaines de l'endroit à la fin du XIX ${ }^{e}$ siècle, a-t-elle laissé dans les collectes des traces identifiables, si minimes soient-elles ? Ce serait chose possible : ainsi, après avoir cru longtemps que la Blanche Biche ne se chantait plus depuis 1937 dans les milieux traditionnels en France, on en a retrouvé un beau fragment dans les archives en 2003, soit la deuxième moitié de la chanson, recueillie dans la Vienne en 1973, enregistrement inclus ${ }^{73}$. La même chose pourrait se reproduire ailleurs, la principale difficulté étant que pour identifier une chanson dans les collectes d'après des fragments, il faut bien la connaître dans son entier et dans ses multiples variantes, et le dépouillement des collectes demande, on le sait, beaucoup de temps et de moyens.

Des aventures de cette chanson, on pourrait d'abord tirer une mise en garde contre les incertaines localisations géographiques des documents provenant des enquêtes de terrain. Marius Barbeau en était éminemment conscient quand il publiait, dans le Journal of American Folk-Lore, sa tentative de retracer les itinéraires des familles des chanteurs de La Tourelle et de Sainte-Anne-des-Monts en Gaspésie-Nord. Voici comment il présentait son article, intitulé « Les premiers colons de Sainte-Anne-des-Monts » :

Comme les débuts de cette localité remontent guère au-delà d'un siècle, la mémoire des octogénaires peut nous servir de guide. La liste suivante des colons, avec indication de leur lieu d'origine et du temps de leur arrivée, démontrera

73. CERDO, coll. Les Gens de Chervès, chanson recueillie auprès de $M^{\text {me }}$ Amicie Pilot, à Ayron dans la Vienne, en 1973. Numérisée et consultable sur le site du CERDO 195.101.116.236/belcerdo/ presentation/basedonnees.htm.sous le titre «Mon fils, retient tes chiens », CERDO (UPCP - Métive Maison des Cultures de Pays 1, rue de la Vau St-Jacques BP03 79201 Parthenay Cedex). 
combien diverse est leur provenance. Gaspé, en ceci, ne ressemble pas aux anciens comtés de Québec, où la souche est stable et relativement homogène. Nous nous en rapportons ici à la mémoire, d'ailleurs faillible, de Pierre Dugas (92 ans), de sa femme Éléanor Lafontaine ( 88 ans), de François Lepage (80 ans) et des membres rassemblés de leurs familles. En eûssions-nous eu le temps, il eût été facile de donner de l'ampleur à notre enquête, d'augmenter ainsi nos renseignements et de corriger les erreurs ${ }^{74}$.

Les itinéraires de cinq de ces familles remontaient jusqu'aux environs de la ville de Québec (Lévis, Beauport, Château-Richer, etc.) ; deux venaient du comté de Charlevoix sur la rive nord du fleuve ; deux étaient d'Acadie, dont une famille issue de la déportation, venue au Québec en passant par Boston. Cinq familles provenaient de l'étranger : deux irlandaises, une jersiaise, une écossaise ; une française « de France ». Les itinéraires de 35 autres familles passaient tous par des localités de la rive sud du Saint-Laurent (comtés de : Bellechasse 4, Montmagny 4, l'Islet 10, Kamouraska 4, Témiscouata 2, Rimouski 9 ; Matane 1).

À supposer que ces familles aient connu leur généalogie en remontant jusqu'aux premiers ancêtres, il est certain qu'on en retrouverait plusieurs dans les environs de Québec (Beauport, Île d'Orléans, Côte de Beaupré, Lévis, etc.) il y a trois ou quatre cents ans, dont les différentes générations de descendants se seraient peu à peu déplacées le long du Saint-Laurent, en «sauts de puce », pour aller s'établir d'une localité à l'autre jusqu'en Gaspésie, glanant au passage des chansons qu'ils ajoutaient à celles de leur propre répertoire. Ainsi, le percheron Charles Pouliot, ancêtre paternel de notre famille Lavoie/Pouliot de Rimouski, s'était d'abord établi à l'île d'Orléans, où l'on pouvait encore admirer la maison ancestrale des Pouliot il y a peu, avant qu'elle ne soit détruite par un incendie.

Quel que soit l'itinéraire suivi par la Blanche Biche de la famille Lavoie/ Pouliot pour arriver jusqu'à elle, cette chanson semble bien avoir eu cours un certain temps dans les milieux franco-américains de Fall-River dans le Massachusetts, entre les années 1888 et 1918, année du retour premier et définitif de Louise-Anna Lavoie à Rimouski dans son pays d'origine. Les allées et venues de cette version sont en tout cas une incitation à se pencher avec beaucoup plus d'attention sur les collections franco-américaines et louisianaises, au cas où s'y seraient coquinement dissimulées, en leur entier ou en fragments, non seulement la complainte qui nous intéresse mais aussi d'autres chansons et contes d'intérêt équivalent.

74. Charles-Marius Barbeau, «Blason, géographie et généalogie populaire de Québec. Les premiers colons de Sainte-Anne-des-Monts », Journal of American Folk-Lore, 1920, n³3, pp. 360-364. 